\title{
Zur Bienenfauna von Abessinien. (Hym.)
}

\author{
Von Dr. H. Friese, Schwerin i. Mecklenburg.
}

Im nachfolgenden bringe ich die erste Zusammenstellung der Bienen (Apidae) von Abessinien, und zwar besonders der Formen aus dem Hochlande bei Harrar (Südost-Abessinien), von woher ich eine reiche Sammlung an Apiden dem Herrn Gunnar Kristensen verdanke, der während einer Reihe von Jahren Nord- und Süd-Abessinien erfolgreich durchforschte. Die ersten Sendungen erhielt ich vor längerer Zeit über Dänemark durch Herrn Jensen-Haarup in Silkeborg aus Nord-Abessinien (Eritrea) von Keren, Ghinda, Asmara und Adua, später direkt von Harrar $(\text { Harar })^{1}$ ) in Südost-Abessinien. Weiter füge ich noch mehrere Arten aus der Umgebung von Abessinien bei, wie z. B. von Somali-Land und dem Sudan, um das Bild zu vervollständigen.

Der geographischen Lage entsprechend finden wir in Abessinien sowohl die tropischen Bienenarten wie auch die letzten Ausläufer der paläarktischen Formen, die offenbar im Hochlande des gebirgigen Abessiniens noch die ihnen zusagenden Bedingungen für ihre Existenz finden. So scheint z. B. noch Halictus virescens Lep., diese häufige und weitverbreitete paläarktische Art, nicht selten vorzukommen, um weiter südlich durch den engverwandten Halictus jucundus $\mathrm{Sm}$. abgelöst zu werden, der dann Afrika bis zum Kapland bewohnt. Ebenso zeigt sich auch der Nomioides pulchelius, diese fast kleinste und sicher zierlichste Bienenart noch häufig in Abessinien, um im Kapland ihre südlichste Verbreitung $\mathrm{zu}$ finden. Auch Anthophora bimaculata und Eriades truncorum, sowie die fast ganz Europa, Asien und Afrika bewohnende Schmarotzerbiene Coelioxys afra Lep. finden sich häufig genug. Neben diesen durchweg gleichgebildeten Formen finden sich aber auch solche, die wohl im Habitus und in der Form den weitverbreiteten paläarktischen Arten gleichen, aber kleinere morphologische Abweichungen aufweisen (vikariierende A rten), wie

Andrena somalica Fr. (für die weitverbreitete A. morio in Südeuropa), Megachile minutissima Rad. (für $M$. rotundata F.),

Epeolus kristenseni Fr. (für E. tristis Sm.),

Andrena africana $\mathrm{F}$. (für $A$. varians $\mathrm{K}$.), Andrena meneliki Fr. (für $A$. floricola Ev.), Andrena kristenseni Fr. (für A. korleviciana Fries.), Halictus meneliki Fr. (für H. smeathmanellus K.),

1) Der Poststempel der Sendungen lautet sowohl Harrar wie Harar, so dafs wohl beide Schreibweisen berechtigt sind. 
Colletes latipes Fr. (für C. rossicus D. T.), Colletes abessinicus Fr. (für C. fodiens Fourc.), Tetralonia femorata Fr. (für T. dentata Klg.), Anthophora ampliceps Fr. (für A. albigena Lep.) und Crocisa histrio F. (für Cr. major Mor.).

Als rein tropische Formen wären dann die Nomia-, Scrapter-, Ceratina- und Xylocopa-Arten zu erwähnen, ferner fast alle Anthophora- und Crocisa-Arten; von den Bauchsammlern sind die meisten Megachile-Arten aufzuführen, und hier besonders die grofsen wie $M$. maxillosa und meneliki, $M$. antinorii und bilobata, combusta und janthoptera wie alle Anthidium-Arten und die bei ersteren schmarotzenden Coelioxys-Formen. -

Die Zahl der neuen Arten, die uns alljährlich aus dem dunklen Erdteil zufliefsen, erdrückt uns fast, die Beschreibungen der nova species nehmen kein Ende und lassen auch keins erhoffen. Dabei erhalten wir fast alle Formen in nur wenigen Exemplaren, weil meist keine Spezialisten und Kenner die Sammler sind - und alle Objekte gelangen fast ohne klärende Notizen über die Umgebung und die natürlichen Verhältnisse, wie Blumenbesuch, Flugzeit, Nestbau usw. in unsern Besitz.

Die früheren Bearbeiter und Autoren hatten keine Ahnung von dem unendlichen Formenreichtum, der uns heute entgegentritt und uns fast $\mathrm{zu}$ erdrücken scheint, sie waren oft $\mathrm{zu}$ sparsam mit den Angaben der wichtigsten Unterscheidungs-Merkmale, sowie der näheren Verwandtschaft zu bekannten Formen. Andererseits mufs auf manche neuere Arbeiten hingewiesen werden, die durch zu grofse Haarspalterei beim Sichten und Unterbringen der Formen - oft sogar noch ohne Angabe der nächststehenden verwandten Spezies - ein Chaos von sogenannten Arten produzieren, deren ungleiche Wertgröfsen als Art das Herausarbeiten des natürlichen Systems ungemein erschweren, wenn nicht unmöglich machen.

Die systematische Spezies umfarst einen Kreis von Formen, die durch ein Hauptmerkmal verbunden sind und zusammengelassen werden müssen, wie wir es bei den hochentwickelten Genera der Bienen, z. B. bei Antophora, Osmia, Megachile und Anthidium so schön ausgeprägt finden. Die Speziesgröfse hat daher innerhalb einer Gattung oder Untergattung annähernd gleichwertig zu sein, so dafs die Formen mit kleineren Abweichungen als Varietäten und Subspezies unterzubringen sind.

Bei einer allgemeinen Betrachtung der Bienen Abessiniens fällt die schwache Vertretung der Urbienen, besonders der Prosopis-Arten auf, die z. B. in Süd-Afrika und auch in Ägypten 
noch viel zahlreicher sind. Ferner fehlen die Vertreter der Panurginae und Melittinae bisher noch ganz, die aus Ägypten und vom Kapland schon genügend bekannt sind. Der Mangel an Osmia-Arten dürfte wohl noch bei genauerer Durchforschung behoben werden, da Ägypten und Kapland zahlreiche Vertreter davon haben, aber das Fehlen der Hummeln (Bombus) trotz des Gebirgslandes mit Höhen bis zu $4600 \mathrm{~m}$ wird wohl bestehen bleiben, da Ägypten, Kilimandjaro und das Kapland auch keine Bombus-Arten aufweisen. Das südlichste Vorkommen von Bombus dürfte Algerien und Marokko (Atlas), Canaren, Sizilien und der Libanon liefern.

Bemerkenswert ist auch noch das Fehler der Schmarotzerbienengattung Nomada, trotz des Vorhandenseins der Wirtbiene Andrena und das nicht seltene Vorkommen der Omachtes-Arten, die offenbar bei Tetralonia oder Anthophora schmarotzen dürften und bisher zu den seltensten Bienen zählten.

Bei den sozialen Bienen ist das Auftreten der Trigona beccarii bei Keren in Eritrea als nördlichster Fundort ( $=16^{0}$ nördl. Br.) erwähnenswert mit dem Hinzufügen, dafs der bisher südlichste Fundort für Trigona in Afrika bei Kooa in der Kalkpfanne (=250 südl. Br.) liegt (für Trigona clypeata Fr.).

Die Zahl der Bienenarten in Abessinien beläuft sich laut Vorliegendem auf 148 Namen, bei einem bisherigen Bestande von über 800 Bienenarten in Afrika südlich der Sahara. Wir haben also wohl kaum die Hälfte der wirklich im abessinischen Gebiete lebenden Arten aufzählen können.

Hoffentlich gestatten uns bald weitere und reichere Sendungen ein tieferes Eindringen in die Fauna und Flora dieses hochinteressanten Gebirgslandes mit seinen ausgedehnten Hochebenen in Höhen von $2000 \mathrm{~m}$ unter dem Tropengürtel in $5-15^{0}$ nördl. Br.

Schwerin, den 1. Februar 1915.

\section{A. Proapidae - Urbienen.}

1. Sphecodes abessinicus n. sp. - 1 ơ $^{7}$ von Harrar ${ }^{1}$ ) im November, Kristensen leg., im Hochland. (Vergl. im Anhang p. 277.) Kleine ganz schwarze Art.

2. Sphecodes eritrinus n. sp. - 1 o von Asmara in Eritrea, Kristensen leg. - Wie Sph. gibbus, aber weifs behaart, vergl. im Anhang p. 277.

1) Die Stadt Harāar oder Harrar = eine Handels-Stadt mit fast 50000 Einwohnern im Lande der Galla. Schreibart auf zweierlei Art, auch der Poststempel lautet bald Harar oder Harrar! 
3. Prosopis gigas Friese (1911). - 1 \& von Ghinda in Eritrea, im Juni fliegend.

4. Prosopis magrettii Vach. - $q$ mehrfach von Keren und Ghinda in Eritrea, im Juni fliegend.

\section{B. Podilegidae - Beinsammler.}

5. Colletes latipes n. sp. - o $q$ mehrfach von Harrar, im November, Kri stens en leg., im Hochland. (Vergl. im Anhang p. 277.) Grofse Art.

6. Colletes abessinicus n. sp. - $\sigma^{7}$ zahlreich bei Harrar im November, Kristensen leg. (Vergl. im Anhang p. 278.) Mittelgrofse Art wie C. fodiens $\mathrm{K}$.

7. Colletes sp. ? - $1 q$ von Asmara im Juni.

8. Halictus weisi n. sp. - $\sigma^{7}+$ mehrfach von Harrar im November, K r istensen leg. (Vergl. im Anhang p. 279.) Gröfsere Art mit weifsgefärbten Segmentbinden.

9. Halictus kristenseni n. sp. - $\sigma^{7}+$ zahlreich von Harrar im November, $\mathrm{Kr}$ istens en leg., $\sigma^{7}$ mehrfach von Ruanda im September in $2700 \mathrm{~m}$ Höhe (Deutsch-Ostafrika). M e y e r leg. (Vergl. im Anhang p. 279.)

10. Halictus abessinicus n. sp. - $\sigma^{7}+$ mehrfach von Harrar, im November fliegend, Kristens en leg., im Hochland. (Vergl. im Anhang p. 280.) Eine Art mit teilweise rotem Abdomen.

11. Halictus flavofasciatus n. sp. - $q$ von Asmara, im Juni fliegend, Eritrea. An den gelbgefärbten Randbinden von Segm. 1-3 zu erkennen. (Vergl. im Anhang p. 280.)

12. Halictus meneliki n. sp. - 2 o $^{7}$ von Harrar, im November, Kristensen leg., im Hochland bei 1200 m. (Vergl. im Anhang p. 281.) Kleine grüne Art.

13. Halictus virescens Lep. - 2 우 von Asmara, im Juni fliegend, Eritrea.

14. Halictus jucundus Sm. - $\sigma^{7}$ 우 von Harrar, im November fliegend, K r is te $\mathrm{n}$ se $\mathrm{n}$ leg., im Hochland bei $1000-1200 \mathrm{~m}$.

15. Halictus (Nomioides) pulchellus Schenck. - Einige $q$ von Asmara, Eritrea. - Kommt auch noch im Kapland bei Willowmore (Brauns) vor, am 5. II. 02 beobachtet.

16. Halictus sp. ? - ca. 3-4 kleinere Halictus-Arten wurden ebenfalls von $\mathrm{Kr}$ is te $\mathrm{nsen}$ gesammelt.

17. Andrena africana Friese (1908). - $\sigma^{7}+$ mehrfach von Harrar, im November fliegend im Hochland, K r is t e n s e n leg. (Vergl. im Anhang p. 281.)

18. Andrena kristenseni n. sp. - $\sigma^{7}+$ einzeln bei Harrar, im November im Hochland fliegend, Kristensen leg. (Vergl. im Anbang p. 282.) 
19. Andrena meneliki n. sp. - $q$ einzeln bei Harrar, im November im Hochland fliegend, $\mathrm{Kr}$ is ten se n leg. (Vergl. im Anhang p. 282.) Eine kleine Art aus der Verwandtschaft von $A$. floricola Ev.

20. Andrena somalica n. sp. - 1 ㅇ vom Somali-Lande, im Mai fliegend. (Vergl. im Anhang p. 283.) Eine grofse, schwarze Art wie A. morio.

21. Nomia parca Kohl (1906). - $\sigma^{7}+$ zahlreich von Harrar, im November fliegend, $\mathrm{Kr}$ istensen leg. Eine kleine, im $\sigma^{7}$ aufserordentlich schmale Art, die von Süd-Arabien beschrieben wurde.

22. Nomia braunsiana Friese (1908). - $\sigma^{7} q$ zahlreich von Harrar, im November gefangen, $\mathrm{Kr}$ istensen leg. Eine kleinere Art mit weifsen Segmentbinden $(2-5)$.

23. Nomia gratiosa Friese (1914). - $\sigma^{7}$ einzeln von Harrar, im November, K r i sten s e n leg. Eine kleine, glänzend schwarze Art, deren Abdominalsegmente vor dem glatten Endrande lang, aber unregelmäfsig weifslich gefranst sind.

24. Nomia magniventris Friese (1914). — $\sigma^{7}+$ mehrfach von Harrar, im November fliegend, Kristen sen leg. Eine mittelgrofse Art mit breiten, blafsgefärbten Segmenträndern und beim $\sigma^{7}$ mit stark bewehrten Beinen III.

25. Nomia vulpina Gerst. - $2 \sigma^{\top}$ von Harrar, im November gefangen, Kristens en leg. Eine weitverbreitete Art, die bis zum Kapland nach Süden geht und am Ukerewe-See massenhaft auftritt.

26. Nomia tridentata Sm. - Einige $\sigma^{7}$ in der Form var. orientalis Fries. bei Harrar im November gefangen; Kriste n s e $\mathrm{n} \mathrm{leg.}$ Weitverbreitete Art, die im Osten, Westen und Süden von Afrika vorkommt.

27. Nomia patellifera Westw. - Einige $\sigma^{7}$ von Harrar, im November fliegend, K ristensen leg. - Teilt die weite Verbreitung mit $N$. tridentata Sm. Das bisher unbekannte $q$ dieser Art vermute ich in der $N$. rotkirchi Friese (1914).

28. Nomia speciosa Friese (1908). - $1 \sigma^{7}$ von Harrar, im November fliegend, $\mathrm{Kr}$ iste $\mathrm{nsen}$ leg., und $3 \sigma^{7}$ von Asmara, im Juli fliegend. - Eine der gröfsten Nomia-Arten und wohl auf Ost-Afrika beschränkt.

29. Nomia somalica Friese (1908). - Einige $q$ von Somali-Land. Eine mittelgrofse Nomia mit rotgelber Abdomenbasis.

30. Nomia friesei Magr. - $\sigma^{\top}$ bei Fra Dimé e il Bass Narok, im August-September fliegend; Eritrea (nach M a g retti).

31. Nomia patellata Magr. - $\sigma^{7}$ von Eritrea (nach Magretti). 
32. Nomia picardii Grib. - $\sigma^{7}$ von Saganeïti in Eritrea. Grofse Art von $15 \mathrm{~mm} \mathrm{~L}$. (nach Gribodo).

33. (?) Nomia quartinae Grib. - $\sigma^{7}$ von Let-Marefiá (Ost-Afrika), - dürfte wohl im Somali-Lande liegen (?).

34. Nomia theryi Grib. - $\sigma^{7}$ von Saganeïti in Eritrea. Grofse Art von $14 \mathrm{~mm} \mathrm{~L}$. (nach Gribodo).

35. Nomia eritrina n. sp. - $\sigma^{7}$ von Asmara in Eritrea, $\mathrm{Kr}$ i st e ns e n leg. (Vergl. im Anhang p. 284.) Eine gröfsere Art aus der Verwandtschaft der $N$. calida Westw.

36. Nomia brevipennis n. sp. - $\sigma^{\top}$ von Harrar, im November, Kristensen leg. (Vergl. im Anhang p. 285.) Eine kleine schwarze Art mit blassen Segmenträndern.

37. Scrapter abessinicus n. sp. $-1 \sigma^{7}$ von Harrar, im Dezember gefangen, Kristensen leg. (Vergl. im Anhang p. 285.) Kleinere, schwarze Art, dem Scr. armatus verwandt.

38. Scrapter nigrotestaceus Magr. - $1 \sigma^{\top}$ von Arussi-Galla. Vergl. Magretti 1895.

39. Scrapter armatus Magr. - $\sigma^{7}+{ }$ von Arussi-Galla (Gamal e Gudda) - 3. Mai 1893, B ot te g o leg. (vergl. Magretti 1895.)

40. Scrapter antinorii Grib. - 1 qon Let-Marefiá (vergl. Gribodo 1884). - $\sigma^{7}+$ mehrfach von Uganda (Britisch-Ostafrika).

41. Ceratina nasalis Friese (1905). - $\sigma^{7}$ 우 von Harrar, im November, Kristensen leg. - Weitverbreitete Art in ganz Ost-Afrika bis zum Kapland, an der blauen Körperfarbe kenntlich.

42. Ceratina lunata Friese (1905). - $\sigma^{7}+9$ mehrfach von Harrar, im November fliegend, K riste $\mathrm{n}$ s en leg. In Ost-Afrika bis zum Nord-Transvaal nicht seltene Art.

43. Allodape candida Sm. - 2 von Harrar, im November gefangen, Kristen sen leg. - Scheint in ganz Ost-Afrika bis zur Delagoa-Bai vorzukommen. 1 으 von Keren (Eritrea), Magretti leg. Häufig am Kilimandjaro im April-Mai, auch auf Marienhof (Insel im Ukerewe-See) häufig, C o n rads leg. Von Marienhof erhielt ich auch ein $\sigma^{7}$, das durch seine ganz abweichende Form (Meliturga-ähnlich) auffällt. (Vergl. im Anhang p. 285.)

44. Allodape foveata Sm. - $\sigma^{7}$ 우 von Harrar, nicht selten im November fliegend, $\mathrm{Kr}$ istensen leg. - Weitverbreitete Art in West- und Ost-Afrika. (o vergl. im Anhang p. 286.)

45. Xylocopa tarsata Sm. - $\sigma^{7}$ 우 mehrfach von Abessinien (Eritrea), 2 or ㅇ von Harrar, im November, K r is te n s e n leg. - Die weitverbreitete Art erhielt ich von Ukami, Kigonsera, Madibira, Dar-es-Salaam, Bukoba. 
46. Xylocopa angolensis Sm. - 2 우 von Eritrea, sonst von Angola und Namaqua-Land (Kap) bekannt geworden; wohl nur eine Form der $X$. tarsata Sm.

47. Xylocopa gaullei Vach. - 1 ○ von Abessinien.

48. Xylocopa villosa Friese (1909). - 2 o7 von Abessinien ohne nähere Fundortsangabe; langpelzig behaarte Art.

49. Xylocopa gribodoi Magr. - 우 으 vom Somali-Land, wo von März--Mai fliegen, ferner von Sheikh Husein, 10. X., von Meo vom 25. X., auch von Usambara, Ikutha und Delalani.

50. Xylocopa erythrina Grib. - Bisher nur o bekannt, von Saganeiti (Eritrea), von Harrar, im November fliegend und auch 3 ๙ von Kigonsera (Deutsch-Ostafrika).

51. Xylocopa longespinosa Enderl. - $1 \sigma^{7}$ von Boran Gallan (SüdGalla), am 2. IV. 01 gefangen.

52. Xylocopa frandulenta Grib. - Mit $X$. carinata die häufigste Holzbiene in Abessinien, im April und Mai fliegend. Auch in Somali-Land und Ost-Afrika bis zum Nyassa-See vorkommend.

53. Xylocopa carinata Sm. - $\sigma^{7}$ ㅇ von Harrar, im November fliegend, $\mathrm{K}$ r is t e $\mathrm{n}$ s e $\mathrm{n}$ leg. - Eine weitverbreitete und nicht seltene Art; von Abessinien über Ost-Afrika bis Salisbury in Mashona-Land, in Natal, Kapland, ferner in West-Afrika von Angola bis Kamerun und Togo. Die Art ändert in Gröfse und Färbung des Haarkleides ab und wurde unter 10 verschiedenen Namen beschrieben.

54. Xylocopa fenestrata F. - $\sigma^{7}$ 을 von Obock (Nordost-Afrika), sehr häufig auf der Insel Réunion, wo sie im Januar fliegt.

55. Xylocopa hottentotta Sm. - o $\sigma^{r}$ von Eritrea, Abessinien, Aden, auch von Jericho (Jordantal) im April, auch in Ägypten.

56. Xylocopa flavorufa Deg. - o sonst auch im ganzen tropischen Afrika nicht selten, auch in Nord-Transvaal noch.

57. Xylocopa flavorufa var. combusta Sm. - $0^{7} \subsetneq$ häufig in Abessinien, so bei Gheleb, Agama, Asmara (Eritrea) im Juni und September fliegend. Sonst in West-Afrika häufiger (Sierra Leone, Kongo, Angola).

58. Xylocopa flavorufa var. kristenseni Friese (1911). selten bei Harrar (1800-1900 m hoch), im November fliegend, $\mathrm{Kr}$ is t e n s e n leg.

59. Xylocopa nigrita Fr. wohl die häufigste Xylocopa-Art in Afrika und überall südlich der Sahara bis Delagoa-Bai und N'Gami im Süden.

60. Xylocopa aestuans L. - $\sigma^{\top} \rightarrow$ dieser weit verbreiteten Art (Afrika und Süd-Asien) auch bei Keren-Asmara in Eritrea 
im September fliegend, auch auf Aden, und im Jordantal (Morice, Schmiedeknecht).

61. Xylocopa erlangeri Enderl. - Einige $q$ von den Galla- und Somali-Ländern im Mai und Juli fliegend.

62. Xylocopa scioënsis Grib. - 1 q von Ambo-Karra (? SomaliLand?); $\sigma^{7}+$ nach Enderlein häufig in Ost-Afrika bis Mosambique und nach Braus noch bei Willowmore (Kapland) im September.

63. Xylocopa somalica. Magr. - $\sigma^{7}+$ aus den Galla- und SomaliLändern, im März fliegend.

64. Xylocopa divisa Klug. - Einige $q$ von Abessinien nach $\mathrm{V}$ a c h a l.

65. Xylocopa inconstans Sm. - o $q$ nicht selten im Süden von Abessinien, ferner im Sudan und am oberen Nil. Die Var. flavocincta Friese (1909) auch von Keren in Eritrea.

66. Xylocopa schoana Enderl. - + aus Schoa, im Oktober und September fliegend; 1 o von Abessinien.

67. Xylocopa taczonovskyi Rad. - sp.? - $\sigma^{7}+$ aus Abessinien (R a f f r a y leg.).

68. Xylocopa nigripes n. sp. $-\sigma^{7}$ 우 nicht selten bei Harrar, im November fliegend, Kristensen leg. (Vergl. im Anhang p. 286.) Kleine, aber auffallend gefärbte Art.

69. Tetralonia fulvicornis Friese (1909). - $\sigma^{7}$ von Gheleb im September (Eritrea).

70. Tetralonia abessinica n. sp. - o $q$ mehrfach von Harrar, im November fliegend, Kristensen leg. (Vergl. im Anhang p. 287.)

71. Tetralonia femorata n. sp. - $\sigma^{7}$ von Harrar, im Oktober, K risten s en leg. - Durch bezahnten Femur III auffallend. (Vergl. im Anhang p. 288.)

72. Anthophora armata Friese (1905). - $\sigma^{7}+$ von Eritrea; der A. semirufa und basalis sehr ähnliche Art und zu den gröfsten Formen der Gattung gehörend.

73. Anthophora armatipes Friese (1911). - $\sigma^{7}$ 우 einzeln von SomaliLand, im Mai fliegend, grofse Art.

74. Anthophora abessinica Friese (1913); olim A. aethiopica. (1911). $2 q$ von Keren (Eritrea), sonst auch in Ost-Afrika bis zum Ukerewe-See verbreitet.

75. Anthophora acraënsis F. - or $q$ einzeln von Keren, im November fliegend.

76. Antophora nubica var. somalica Magr. - + von Somali-Land und Kongo.

77. Anthophora plumipes F. - Diese fast über ganz Afrika verbreitete Art, kommt auch in Eritrea vor, wo $2 q$ bei Mei Hadega, 5. II. gefangen wurden, Mag retti leg. 
78. Anthophora circulata F. - 1 q von Eritrea, sonst weit verbreitete und häufige Art, besonders im Kapland.

79. Anthophora ampliceps var. kristenseni n. var. - Einige $\sigma^{7}$ von Harrar, im November fliegend, Kristensen leg. (Vergl. im Anhang p. 288.)

80. Anthophora eritrina n. sp. - or o mehrfach von Asmara, im Juni fliegend; 1 q von Mulango (Brit.-Ostafrika) und 2 व 1 의 von Zungarn (N.-Nigeria), im November 1910, im Mus. London. (Vergl. im Anhang p. 289.)

81. Anthophora flaviventris n. sp. - $2 \sigma^{7}$ von Asmara (Eritrea), im August fliegend. (Vergl. im Anhang p. 289.)

82. Anthophora capensis Friese (1905). - 1 우 von Asmara (Eritrea), im Juli fliegend.

83. Anthophora nigrifaceis Friese (1905). - 1 o $\sigma^{7}$ von Eritrea.

84. Anthophora caerulea Friese (1905). - 우 우 nicht selten bei Harrar, im November fliegend, Kristensen leg. (Vergl. im Anhang p. 290.) Auch bei Gheleb in Eritrea, im September. Sonst im ganzen tropischen Afrika bis Natal (1 우 im Kap-Museum).

85. Anthophora cinerascens Lep. - + aus Nubien.

86. Anthophora senescens Lep. - o Süd-Europa und Ägypten, 1 q von Eritrea (Asmara).

87. Anthophora crocea Klug. - 1 aus Sokotra; sonst aus Ägypten bekannt.

88. Anthophora bimaculata Panz. - $\sigma^{\top}$ aus Eritrea, sonst nur im paläarktischen Gebiet.

89. Anthophora byssina Klug. - 1 ㅇ von Eritrea, sonst in Ägypten heimisch.

90. Anthophora rufipes Lep. - + aus Kaffernland, $\sigma^{\top}$ aus Abessinien (vergl. D o u r s), nach D o u r s = Anthoph. rufa Savig. Lep.

91. Anthophora tuberculilabris Dours. - 2 우 von Abessinien nach Dours.

\section{1. Schmarotzerbienen: Melectinae.}

92. Crocisa calceata Vach. - $\sigma^{7} \nmid$ häufig bei Harrar, im November, $\mathrm{Kr}$ istensen leg. Die Zeichnungen sind fast weils mit bläulichem Schein.

93. Crocisa meripes Vach. - 1 ㅇ von Gheleb in Eritrea; auch bei diesem Exemplar sind die hellen Zeichnungen fast weifs, statt bläulich.

94. Crocisa histrio F. - $\sigma^{r}$ von Nubien und Eritrea, im November fliegend; 1 o von Keren, im Februar durch $\mathbf{M}$ a gretti beobachtet; 1 o von Ghinda, im Mai. 
95. Crocisa hyalinata Vach. - $1 \sigma^{7}$ von Asmara (Eritrea), im Juni gefangen.

96. Crocisa abyssinica Rad. - $q$ von Ägypten und Abessinien, Spezies? und unsicher.

97. Crocisa forbesii Kirby. - $\sigma^{7}+$ von Sokotra, nach $\mathrm{Kohl}=$ eine Nomia scutellaris var.

98. Crocisa uniformis Kirby. - $q$ von Sokotra; nach Kohl der Cr. ramosa verwandt, aber gröfser und ganz dunkel, fast einfarbig.

99. Epeolus kristenseni n. sp. - $\sigma^{7}+$ mehrfach von Harrar, im November fliegend, $\mathrm{Kr}$ is te $\mathrm{n}$ s en leg. Schmarotzt wahrscheinlich bei Colletes abessinicus Friese. (Vergl. im Anhang p. 290.)

\section{Gastrilegidae - Bauchsammler.}

100. Eriades argentatus Gerst. - $\sigma^{7}$ von Ghinda in Eritrea, im Juni fliegend.

101. Eriades filicornis n. sp. - $2 \sigma^{7}$ von Harrar, im November fliegend, Kristensen leg. (Vergl. im Anhang p. 291.)

102. Eriades abessinicus n. sp. $-2 \sigma^{7}$ von Harrar, im November gefangen, Kristensen leg. (Vergl. im Anhang p. 292.)

103. Eriades truncorum L. - $3 \sigma^{7}$ von Harrar, im November fliegend, Kristensen leg. (Vergl. im Anhang p. 292.)

104. Megachile maxillosa Guer. - ơ + vom Webi-Tal (SomaliLand), von Kartum (Sudan), Abessinien; fliegt an Acacia horrida.

105. Megachile bicornuta Friese (1903). - $q$ von Kartum (Sudan). 106. Megachile mephistophelica Grib. - $q$ von Kassala (Nubien).

107. Megachile sokotrana Friese (1903). - + von Sokotra, im Januar fliegend.

108. Megachile simonyi Friese (1903). - $\sigma^{7}$ 우 von Keren (Eritrea), im Februar fliegend, M a g r etti leg.

109. Megachile rufiventris Guer. - $\sigma^{7}+9$ von Eritrea.

110. Megachile grandiceps Friese. - $q$ von Keren (Eritrea).

111. Megachile antinorii Grib. - $\sigma^{\top}+$ nicht selten in Abessinien und im Sudan; grofse Art mit rotfilzigem Abdomen.

112. Megachile bilobata n. sp. - Einige or o von Harrar, im November fliegend, Kristensen leg. (Vergl. im Anhang p. 292.)

113. Megachile meneliki n. sp. $-2 q$ von Süd-Abessinien. Grölste Megachile-Art von Afrika (L. 24-25 mm, Br. $8 \mathrm{~mm}$ ). (Vergl. im Anhang p. 293.)

114. Megachile quadrispinosa Friese (1904). - $2 \sigma^{7}$ von Nubien. 115. Megachile kerenensis Friese (1904). - 2 q von Keren (Eritrea), im Februar fliegend, Magretti leg., $4 q$ von Eritrea. 
116. Megachile combusta Sm. - $\sigma^{\top}+q_{\text {von Eritrea und } 1}+$ von Harrar, im November fliegend, K r is te n s e n leg.

117. Megachile janthoptera Sm. - $1 \sigma^{\top}$ von Harrar, im November gefangen, K rist en sen leg., sonst im Süden eine häufige Biene.

118. Megachile cognata Sm. - Einige of von Eritrea, ohne besondere Fundortsangabe.

119. Megachile abessinica n. sp. - or 우 mehrfach von Harrar, im November fliegend, K ristensen leg. (Vergl. im Anhang p. 293.)

120. Megachile harrarensis n. sp. $-2 \sigma^{7}$ von Harrar, im November fliegend, $\mathrm{Kr}$ istensen leg. (Vergl. im Anhang p. 294.)

121. Megachile fülleborni Friese (1903). - $\sigma^{7}$ 우 einzeln in Abessinien, die Art dürfte identisch mit M. sudanica Magr. (1899) sein.

122. Megachile patellimana Spin. - $\sigma^{7}+$ aus Ägypten, $\sigma^{7}$ auch von Harkeko (am Roten Meer).

123. Megachile albocincta Rad. - $\sigma^{7}+$ von Ägypten, Luxor-Kartum, auch im Sudan.

124. Megachile aduaënsis Friese (1909). - $q$ von Eritrea, $\mathrm{K}$ ristensen leg.

125. Megachile chelostomoïdes Grib. - $\sigma^{\top}$ von Saganeiti in Eritrea. 126. Megachile colorata Fox. -2 aus Somali-Land.

127. Megachile crenulata Fox. - $\sigma^{7}$ von Sheik Husein, im September, Somali-Land.

128. Megachile galactogagates Grib. - + von Saganeiti in Eritrea. 129. Megachile saganeitana Grib. - $\sigma^{7}$ von Saganeiti in Eritrea. 130. Megachile marchalli Friese (1904). - + von Eritrea, im November fliegend.

131. Megachile minutissima Rad. - $\sigma^{7}+$ von Keren in Eritrea; sonst in Ägypten häufiger.

132. Anthidium cordatum var. mefescens n. var. $-\sigma^{\top} q$ von Harrar, im November gefangen, Kristensen leg. (Vergl. im Anhang p. 294.)

133. Anthidium eritrinum n. sp. - 1 q von Gheleb im September, Kristensen leg., Eritrea. (Vergl. im Anhang p. 295.) 134. Anthidium sjöstedti Friese (1908). - $2 \sigma^{7}$ von Harrar, im November fliegend, Kristensen leg. - Die Art wurde von Prof. Sjös te dt am Kilimandjaro entdeckt, darauf auch von Deutsch-Ostafrika nachgewiesen (Lukuledi) und nun auch von Südost-Abessinien. A. sjöstedti fehlt in meinem zusammenfassenden Werk "Die Bienen Afrikas" (Jena 1909), sie wurde versehentlich fortgelassen und ist hier p. 406 hinter $A$. truncatum Sm. einzuschalten. 
135. Anthidium junodi Friese (1904). - 1 q von Harrar, im November fliegend, Kristensen leg. Die Art wurde bisher nur von Süd-Afrika nachgewiesen, so von Transvaal, Port Elizabet, Willowmore, Kapstadt und hier vom Dezember bis April fliegend.

\section{2. Schmarotzerbienen : Coelioxyncue.}

136. Coelioxys scioënsis Grib. (1879). - ot우 mehrfach von Harrar, im November gefangen, Kristensen leg. - Auch von Eritrea und dem Sudan, ferner von Guinea, Nyassa und Usambara vorliegend.

Dürfte bei Megachile antinorii schmarotzen.

137. Coelioxys decipiens Spin. - Weitverbreitete Art von Ägypten und Abessinien nicht selten erhalten, auch in Süd-Europa - Mittelasien und Ost-Afrika bis Transvaal verbreitet.

138. Coelioxys cherenensis Friese (1913). - $\sigma^{7}+$ mehrfach von Cheren (Keren) in Eritrea, 1 sehr grofses $q$ aus dem Sudan.

139. Coelioxys somalina Magretti - $\sigma^{\top}$ von Galla-Ländern.

140. Coelioxys magrettii n. n. (= obtusata Magretti) - $\sigma^{\top}$ von Ganale Gudda (Galla-Länder). Da C. obtusata bereits von $\mathrm{Sch}$ enck (1855) vergeben wurde, so ist hier die Namensänderung notwendig.

141. Coelioxys afra Lep. - $1 \sigma^{\top}$ von Harrar, im November, Kristensen leg. - Weitverbreitete Art. - Europa Afrika - Asien.

142. Omachtes nigripes n. sp. - Einige or o von Eritrea, auch 1 i von Usambara. (Vergl. im Anhang p. 296.)

143. Omachtes abessinicus n. sp. - 1 q von Eritrea. (Vergl. im Anhang p. 297.)

\section{E. Soziale Apiden.}

144. Trigona beccarii Grib. - 9 von Keren in Eritrea, beschrieben, mir liegen auch nur $P$ aus Eritrea vor.

145. Trigona armata Magr. - $\sigma^{7}$ von Arussi-Galla (Ganale Gudda). 146. Trigona bottegoi Magr. - Bisher im 9 von Arussi-Galla und Ganale Gudda vorliegend, 19 auch von Bulawayo, 5. X. 13, A r nold leg.

147. Trigona ruspolii Magr. - 9 zahlreich von „Dana el l'Uebi, qualcuno a Dolo" (? Galla-Länder).

148. Apis mellifica var. adansonii Latr. - Einzelne 9 von Harrar, im November gefangen, $\mathrm{Kr}$ is tensen leg., sonst vom Somali-Lande und Galla-Lande bekannt geworden, wie im tropischen Afrika. 


\section{Anhang.}

Zu Nr. 1. Sphecodes abessinicus n. sp. or.

Sph. abessinicus fällt durch die schwarze Farbe seines ganzen Körpers auf.

$\sigma^{7}$. Schwarz, stellenweise kurz weifs behaart, Kopf und Thorax sehr grob runzlig punktiert, glänzend, Kopf, Ränder des Mesonotums und Tegulae schwach weifs behaart; Antenne dick, knotig und ganz schwarz, Scutellum wulstig erhaben; Area grob längsrunzlig, scharf kielig gerandet, Umgebung grob und höckrig gerunzelt. Abdomen fast glatt, stark glänzend, nur Segment 6-7 mit einzelnen weifsen Haaren. Ventralsegmente mit braunen Endrändern, kahl. Beine schwarz, mit einzelnen weifsen Haaren, Calcar schwarz. Flügel hyalin, mit dunkler Endhälfte, Adern dunkelbraun, Tegulae schwarz. L. $5^{1 / 2} \mathrm{~mm}$, Br. $1^{1 / 2} \mathrm{~mm}$.

$1 \sigma^{\top}$ von Harrar, im November, Kristensen leg. im Hochland.

Südost-Abessinien.

$\mathrm{Zu}$ Nr. 2. Sphecodes eritrinus n. sp. $\quad$ q.

Wie Sph. gibbus, aber ziemlich dicht weifs behaart, Segment $1-3$ rot, $4-6$ schwarz.

․ Schwarz, lang behaart, Kopf und Thorax dicht runzlig punktiert, wenig glänzend, Clypeus grob punktiert, Mandibel fast rot, Gesicht dicht weifs behaart, glänzend, Antenne sehr kurz, schwarz, 2. Geifselglied $=3$. Mesonotum und Scutellum grob punktiert, etwas glänzend; Thoraxseiten, Metanotum und Tegulae dicht weifsfilzig, Area sehr grob und höckrig gerunzelt, glänzend. Abdomen punktiert, mit glatten Segmenträndern. Segment $1-3$ rot, Segmentrand von 1 auch punktiert, 4-6 schwarz, unregelmäfsig punktiert, Segment 1 und Seiten von 2-5 mit weifsen Haaren, 5 mitten und 6 ganz schwarzbraun behaart. Ventralsegment 1-4 rotgelb, weifslich behaart, $5-6$ schwarzbraun, 5 weifs behaart und 6 schwarzbraun behaart. Beine schwarz, Tibie I und Tarsen braun, alles weifs behaart, Scopa rein weifs, Calcar rotgelb. Flügel fast hyalin, mit schmalem, braunem Rande, Adern und Tegulae hellgelb. L. $10 \mathrm{~mm}$, Br. $3 \mathrm{~mm}$.

$1 q$ von Asmara in Eritrea, 1908, K r i s te $\mathrm{n}$ s e $\mathrm{n}$ leg.

Sph. eritrinus fällt vor allem durch die weifse Behaarung auf, wie sie bisher bei Sphecodes o nur bei der Steppenform Sph. rufithorax Mor. aus Turkestan beobachtet wurde, die aber roten Thorax und rote Beine hat.

5. Colletes latipes n. sp. oro.

Wie C. rossicus. D. T. (= fasciatus Rad., grandis Friese) aus 
Ägypten, aber Abdomen infolge feinster Runzelung ganz matt, Area grob gerunzelt, $q$ Beine schwarz.

․ Schwarz, lang gelbbraun behaart, Kopf und Thorax fein punktiert, etwas glänzend, Clypeus verlängert, längsrunzlig, glänzend; Wangen lang, fast quadratisch, meist glatt; Antenne schwarz, 2. Geifselglied $=3 .+4$. Mesonotum ziemlich grob, aber flach punktiert, glänzend, mit fast glatter Scheibe, Area grob gerunzelt, glänzend und stark gegen die äufserst fein gerunzelte und matte Umgebung abstechend. Abdomen schwarz, mit gelblichen Segmenträndern, äufserst fein gerunzelt, matt, nur auf Segment 1 mit feinen erkennbaren Punkten, Segment 1-2 nur seitlich mit breiten Filzbinden, 3-5 mit ganzen, aber zarten Filzbinden am Endrande, 6 schwarz behaart. Ventralsegmente fein und undeutlich punktiert, glänzend, dabei fein quergerunzelt, gelblich gefranst. Beine schwarz, gelblich behaart, Scopa dünn und Calcar gelblich, Metatarsus nur wenig schmaler als die Tibie, 2. Tarsenglied so breit wie 1. Flügel gelblich getrübt, Adern rotgelb, Tegulae gelbbraun. L. $14-15 \mathrm{~mm}$, Br. $4^{1 / 2} \mathrm{~mm}$.

$\sigma^{7}$ wie $q$, aber Kopf verlängert, Wangen länger als breit, Antenne länger, erreicht das Thoraxende, 2. Geifselglied $=3$.; Segment 1-2 so lang und dicht gelbbraun wie der Thorax behaart, Segment 2-5 mit dünnen, weifslichen Filzbinden, 6-7 dunkel. Ventralsegmente flach, fast eingedrückt, kahl, 6 mit breitem, rotem Endrand, der mitten vorgezogen ist, Basis mit kleinem, kurzborstigem Felde. Beine kurz und stark verdickt, Femur keulenförmig, Tibie verdickt mit scharfer Aufsenkante, Tarsen verkürzt und sehr breit, Metatarsus (III) von Tibienbreite und quadratisch, Glied 2-4 nach hinten lappig erweitert, auch bei den Beinpaaren I und II, aber in geringerem Mafse. L. $13 \mathrm{~mm}$, Br. $4 \mathrm{~mm}$.

$\sigma^{7}+9$ mehrfach von Harrar, im November, $\mathrm{K}$ r i s te $\mathrm{n}$ s e $\mathrm{n}$ leg. Ost-Abessinien - Hochland.

6. Colletes abessinicus n. sp. or.

Dem C. rufitarsis Fr. von Ost-Afrika sehr nahestehend, aber kräftiger gebaut, Abdomen grob und gestochen punktiert und Beine schwarz.

$\sigma^{7}$. Schwarz, dicht und lang gelbbraun behaart, Kopf und Thorax grob und runzlig punktiert, glänzend, Wangen wie bei C. ruftarsis, Antenne schwarz, 2. Geifselglied viel kürzer als 3., das 3. $=4$. Mesonotum-Scheibe einzeln und sehr grob punktiert, Area wie bei C. rufitarsis gerunzelt, etwas gröber und stark glänzend, Abdomen grob punktiert, glänzend, Segment 1-2 aber nicht gröber, Segmentränder schwarz, fast glatt, 1 mit schmaler, 
2-5 mit breiter gelblichweifser Filzbinde, 6-7 dunkel behaart. Ventralsegmente flacher punktiert, glänzend, lang gelblich gefranst. Beine schwarz, nur Klauenglied rotgelb, Calcar gelbbraun, sonst sparsam weifslich behaart. Flügel getrübt, Adern und Tegulae rotgelb. L. $10 \mathrm{~mm}, \mathrm{Br} .3^{1 / 2}-4 \mathrm{~mm}$.

$\sigma^{7}$ zahlreich bei Harrar im November, Kristensen leg. Ost-Abessinien.

\section{Halictus weisi n. sp. ơ?.}

H. weisi fällt durch die grünlich weifsen Randbinden von Segment 1-4 (-5) auf, wie sie bei Nomia-Arten vorkommen, und gehört in die Verwandtschaft des $H$. albofasciatus $\mathrm{Sm}$. und $H$. nomioides Fries.

9. Schwach gelblich behaart, Kopf und Thorax dicht runzlig punktiert, matt; Gesicht fein längsrissig, Clypeus vorgezogen, mit einzelnen grofsen Punkten, am Endrand gewulstet mit gröberen und dichtstehenden Punkten; Antenne braun, 2. Geifselglied so lang wie das 3. Mesonotumscheibe mit einzelnen flachen Punkten, seitlich dichter und grob runzlich punktiert, Area fast horizontal liegend, scharfkantig umrandet, hinten mit 2 stumpfen Ecken und längsrunzlig. Abdomen äufserst fein skulpturiert, Segment 1-4 mit grünlich- bis bläulichweifs gefärbten, ziemlich breiten Segmentbinden am Endrande, 5-6 schwarzbraun behaart. Ventralsegmente ganz matt, fein gerunzelt. Beine schwarz, Tarsen braun, schwarzbraun behaart, Scopa fast schwarz. Flügel gelblich getrübt, Adern und Tegulae schwarzbraun. L. $91 / 2-10 \mathrm{~mm}$, Br. $3 \mathrm{~mm}$.

$\sigma^{7}$ wie + , aber Clypeusendrand und Labrum gelb, Segment 1-5 mit fast weifsen Segmentbinden, 6-7 schwarzbraun; Ventralsegmente $3-4$ breit ausgerandet. L. $9 \mathrm{~mm}$, Br. $2^{1 / 4} \mathrm{~mm}$. $\sigma^{7}+$ mehrfach von Harrar, im November fliegend, Kriste nse $n$ leg.

Südost-Abessinien.

9. Halictus kristenseni n. sp. 우우.

$H$. kristenseni ist dem $H$. weisi täuschend ähnlich, aber viel kleiner, Kopf und Thorax gleichmälsig gerunzelt und matt, $\sigma^{\top}$ mit schwarzem Clypeus und Labrum.

․ Schwarz, gelblich behaart, Kopf und Thorax dicht runzlig punktiert, matt, wie H. weisi, aber Mesonotum ohne einzelne, gröfsere Punkte, Abdomen punktiert, Segmentendhälfte feiner, Randbinden sehr schmal, Segment 5-6 schwarzbraun behaart. Ventralsegmente mit glatter, glänzender Basis. Beine schwarzbraun behaart, Tibie III am Ende und unten, sowie die Basis des Meta- 
tarsus rotgelb, Scopa oben schwarzbraun, unten weifslich, am Ende rotgelb, Calcar gelblich. Flügel getrübt, mit dunklerem Rande, Adern braun, Tegulae glänzend schwarz. L. $8 \mathrm{~mm}$, Br. $2 \mathrm{~mm}$.

$\sigma^{7}$ wie $q$, auch Clypeus und Labrum schwarz, Antenne gekerbt erscheinend, etwas plattgedrückt, 2. Geifselglied kürzer als 3. Segment 1-5 sehr schmal, fast linear gelb bandiert; Ventralsegment glatt und glänzend, 3-4 tief bogig ausgerandet. Beine fast einfarbig schwarz bis schwarzbraun, nur Tarsenglied (2) $3-5$ braun. L. $7 \mathrm{~mm}, \mathrm{Br} .13 / 4 \mathrm{~mm}$.

$\sigma^{7}+$ zahlreich von Harrar, im November, $\mathrm{K}$ r i s t e n s e $\mathrm{n}$ leg. Südost-Abessinien, Hochland, und $2 \sigma^{7}$ von Ruanda, am 7. September, im 2700 m Höhe (Deutsch-Ostafrika), M e y e r leg.

10. Halictus abessinicus n. sp. or우.

H. abessinicus ist dem $H$. weisi nahestehend, aber Segment 1 oder 1-2 rot gefärbt, $\sigma^{\top}$ mit eigenartig bewehrtem Ventralsegment 4.

9. Schwarz, gelblich behaart, Kopf und Thorax dicht runzlig punktiert, ganz matt, Clypeus gewölbt, vorgezogen, glänzend und mit groben Punkten, die erkennbaren Punkte des Mesonotum sehr undeutlich, Area gerunzelt, aber kaum noch längsrunzlig. Abdomen fein und undeutlich skulptiert, mit schwach erkennbaren Punkten, Segment 1 rot, oft auch Basis von Segment 2, Segment 1-4 mit breit weifsgefärbtem Endrand, 5-6 schwarzbraun behaart. Ventralsegmente mit einzelnen ziemlich groben haartragenden Punkten, lang weifslich gefranst. Beine schwarzbraun, braun behaart, Scopa oben schwarzbraun, unten weifslich, Calcar rotgelb. Flügel stark getrübt, mit dunklem Endrand, Adern und die glänzenden Tegulae schwarz. L. $8-9 \mathrm{~mm}, \mathrm{Br} .2 \mathrm{~mm}$.

$\sigma^{7}$ wie + , aber Clypeus und Labrum gelb, Segment 1 rötlich, 2-3 braun, 1-5 mit schmalerem, weifsem Endrande, 6 schwarz, 7 gerundet und rot. Ventralsegmente $1-4$ gelbbraun, 4 schmal, an der Basis mit breitem, mitten gefurchtem Höcker, am Endrand jederseits mit $9-10$ rotgelben Wimperborsten, die gewinkelt sind und nach unten abstehen, 5-6 schwarzbraun. L. $8 \mathrm{~mm}, \mathrm{Br}$. $7^{3} / 4 \mathrm{~mm}$.

$\sigma^{7}++$ mehrfach von Harrar, im November, K r i s t e $\mathrm{n}$ s e $\mathrm{n}$ leg. Südost-Abessinien, Hochland.

11. Halictus flavofasciatus n. sp. ‥

Dem H. albofasciatus Sm. vom Kapland ähnlich, aber Segment 1-3 mit breiteren und gelbgefärbten Binden am Endrande.

․ Schwarz, kurz gelbbraun behaart, Kopf und Thorax sehr 
fein runzlig punktiert, Wangen deutlich, Clypeus etwas verlängert und grob punktiert, Gesicht gröber gerunzelt; Antenne und Mandibel schwarz; Mesonotum und Scutellum äufserst fein gerunzelt, matt, mit einzelnen grofsen flachen Punkten; Area hinten eckig und scharf gerandet, mit Querrunzeln. Abdomen schwarz, fein runzlig punktiert, wenig glänzend, Segment 1 sehr fein punktiert, die niedergedrückten Endränder der Segmente 1-3 breit gelb gefärbt, fast matt, $1-3$ sonst gelblich behaart, $4-6$ schwarzbraun behaart, 4 mit braunem Endrand; Ventralsegment 1-3 mehr weniger rotgelb, 4-6 schwarzbraun, alle fein gerunzelt und auf dem Basalteil mit deutlichen Punkten, gelblich gefranst. Beine schwarzbraun, Femur gelblich behaart, Tibien und Tarsen schwarzbraun behaart, Calcar rotgelb, Tibie II unten an der Basis mit rotgelbem Borstenbüschel, Tarsenglied 2-5 rotbraun. Flügel getrübt, mit gleichartigem Rande, Adern und Tegulae braun. L. $10 \mathrm{~mm}$, Br. $3 \mathrm{~mm}$.

Nur $q$ von Asmara, im Juni fliegend, Kristensen leg. Eritrea.

Nord-Abessinien.

Im Habitus an das Subgen. Trinchostoma Sauss. erinnernd, aber anderes Flügelgeäder.

\section{Halictus meneliki n. sp. or.}

Dem H. smeathmanellus K. sehr ähnlich, etwas kleiner, Clypeus ohne gelben Endrand, Area längsrissig, nicht längsrunzlig.

$\sigma^{7}$. Erzgrün, sparsam weifs behaart, Kopf dicht runzlig punktiert, matt, Stirnschildchen fast glatt, glänzend mit einzelnen Punkten, Clypeus verlängert, abgestutzt. Mesonotum und Scutellum fein punktiert, glänzend, Area fein längsrissig. Abdomen gleichmäfsig fein punktiert, glänzend, Segment 6-7 mit einzelnen, weilsen Haaren; Ventralsegmente punktiert, mit glatten, braunen Segmenträndern Beine schwarz bis schwarzbraun, Calcar weifs, sonst weifs behaart. Flügel hyalin, Adern und Tegulae braun. L. $4-4^{1} / 2 \mathrm{~mm}$, Br. $1 \mathrm{~mm}$.

$2 \sigma^{\top}$ von Harrar, im November, Kristens e n leg., Hochland von $1200 \mathrm{~m}$.

Südost-Abessinien.

17. Andrena africana Friese. $\sigma^{7}$.

1913. A. abessinica Friese i. 1. - $\sigma^{7}-$

$\sigma^{7}$ wie $q$ und $\operatorname{dem} A$. varians K. $\sigma^{7}$ in der Form ähnlich, resp. der $A$. fuscipes K. infolge der Abdomenbehaarung; Kopf und Thorax sehr fein runzlig punktiert, matt; Arèa des Mittelsegments feiner als die Umgebung skulptiert. Antenne schwarz, 2. Geifsel-

Deutsche Entomol. Zeitschrift 1915. Heft III. 
glied fast so lang wie $3+4$. Abdomen fein und sehr dicht runzlig punktiert, fast quergerunzelt und mit schwachem Erzschimmer, der auch beim o mitunter sichtbar wird, Segmente lang behaart, aber kaum Binden bildend, 6-7 rötlich behaart. Ventralsegment deutlich querrissig, mit einzelnen feinen Punkten. Beine schwarz, gelblich behaart, Calcar gelblich. Flügel wie beim o "schwach" getrübt (nicht $\mathrm{schwarz}$, wie in der Originalbeschreibung gedruckt wurde!), Adern gelblich, Tegulae braun. L. $7 \frac{1}{2}-8 \mathrm{~mm}$, Br. $2 \mathrm{~mm}$.

$\sigma^{7}+$ mehrfach von Harrar, im November fliegend, K r iste n s e n leg., Hochland von $1200 \mathrm{~m}$.

Südost-Abessinien.

18. Andrenakristenseni n. sp. 주웅.

Der A. korleviciana Fr. von Fiume sehr ähnlich, aber Abdomen sehr fein gerunzelt, matt, mit einzelnen feinen Punkten und Segmentbinden undeutlich, fein und unterbrochen.

․ Schwarz, lang gelbbraun behaart, Kopf und Thorax sehr fein gerunzelt, Clypeus gröber und runzlig punktiert, alles matt; Antenne schwarz; Mesonotum gröber und runzlig punktiert; Area gerunzelt und nicht von der Umgebung abstechend. Abdomen sehr fein gerunzelt, ganz matt, mit einzelnen, feinen Punkten, Segment 1 einzeln lang behaart, 2-4 seitlich mit breiten aber sehr schwachen gelblichen Fransenbinden, 6 rotgelb beborstet. Ventralsegmente einzeln und grob flach punktiert, gelblich gefranst, 6 ganz rotgelb behaart, 4-6 flach eingedrückt und hier fast glatt. Beine schwarz, ziemlich lang gelblich behaart, Calcar rotgelb, Scopa rotgelb, Metatarsus breit, fast von Tibienbreite. Flügel schwach getrübt, besonders am Endrande, Adern gelbbraun, Tegulae rotgelb. L. $10 \mathrm{~mm}$, Br. $3 \mathrm{~mm}$, Abdomenbreite $3 \frac{1}{2} \mathrm{~mm}$. $\sigma^{7}$ wie $q$, Abdomen wie der Thorax lang gelbbraun behaart (wie A. fulva Christ $\sigma^{\top}$ in Mitteleuropa), Segmentbinden nicht erkennbar. Beine schwarz, gelbbraun behaart; Ventralsegment 4 glatt, glänzend, mit breitem Basalhöcker und Querfurche dahinter, 5 glatt, 6 gerunzelt. L. $9 \mathrm{~mm}, \mathrm{Br} .2^{1 / 1} \mathrm{~mm}$.

$\sigma^{7}+9$ einzeln von Harrar, im November fliegend, Kristensen leg., Hochland von $1200 \mathrm{~m}$.

Südost-Abessinien.

19. Andrena meneliki n. sp. +.

Der A. floricola Ev. äufserlich sehr ähnlich, aber gröfser, Area fein längsrissig, Abdomen ohne gröfsere Punkte.

9. Schwarz, gelbbraun behaart, Kopf und Thorax dicht und fein gerunzelt, matt, Kopf viel breiter als lang, Clypeus zurück- 
gedrängt, glatter, mit einzelnen erkennbaren Punkten in der Runzlung; Antenne schwarz, unten schwarzbraun; Area fein längsrissig, nur schwach gegen die Umgebung abstechend. Abdomen fast unbehaart, schwarz, undeutlich, aber einheitlich punktiert, so dafs Basal- und Endrand nicht voneinander abstehen, Segment 6 rotgelb gefärbt, 5 (Endhälfte) und 6 rotgelb behaart. Ventralsegmente fein gerunzelt, dem Endrande $\mathrm{zu}$ mit haartragenden Punkten, 3-5 mitten etwas eingedrückt. Beine schwarz, gelbbraun behaart, Calcar rotgelb, Metatarsus braun. Flügel fast hyalin, Adern braun, Tegulae schwarz. L. $8 \mathrm{~mm}, \mathrm{Br} .2^{1} / 2 \mathrm{~mm}$.

2 o von Harrar, im November, Kristensen leg., Hochland von $1000 \mathrm{~m}$.

Südost-Abessinien.

20. Andrena somalica n. sp. q.

Wie A. morio Br. aus Süd-Europa, aber Mesonotum und Scutellum kahl, fast glatt mit wenigen flachen Punkten, glänzend, Clypeus verkürzt, Mandibel rotbraun.

․ Schwarz, sparsam schwarz behaart, oben fast kahl, Kopf äufserst fein gerunzelt, matt mit einzelnen groben Punkten, Clypeus kurz, die unteren Augenränder nicht überragend, fast abgestutzt, runzlig punktiert, Wangen linear, rotgelb, Mandibel rotbraun, mit schwarzem Ende; Antenne schwarzbraun, Schaftende und Basis, sowie Unterseite der Geifsel rotbraun, 2. Geifselglied länger als $3+4,3$. und 4. unten rotgelb. Mesonotum und Scutellum fast glatt, glänzend, mit einigen grofen Punkten, Metanotum wie der übrige Thorax runzlig punktiert, Area viel feiner und matt. Abdomen fast kahl, sparsam und schräg gestochen punktiert, ziemlich glänzend. Segment 5 und 6 schwarzbraun behaart; Ventralsegmente runzlig punktiert, Segmente dicht bürstenartig behaart. Beine schwarz, schwarz behaart, Scopa dicht, kurz und schwarz, Calcar rotgelb. Flügel schwarzbraun, blau schimmernd, Adern und Tegulae braun. L. $17-18 \mathrm{~mm}$, Br. $5^{1 / 2}$ - des Abdomen $6 \mathrm{~mm}$.

1 q vom Somali-Lande, im Mai fliegend.

Nordost-Afrika.

A. somalica ist eine bemerkenswerte Form, da sie uns das südlichste Vorkommen der grofsen Andrena-Arten kundtut, die sonst nur dem paläarktischen Gebiet angehören. Als südlichster Fundort für Andrena ist der Kilimandjaro bekannt geworden, wo die Andrena africana noch fliegt, die aber laut vorliegendem Ergebnis auch bei Harrar mit Andrena kristenseni und A. meneliki gefunden wurde.

(Grölste, d. h. breiteste Andrena-Art!) 
35. Nomia eritrina n. sp. or.

Der $N$. calida Westw. sehr ähnlich, aber Antenne und Beine schwarz, Segment 2-5 mit gelben Binden.

๙7. Schwarz, gelbbraun behaart, Kopf und Thorax dicht runzlig punktiert, ganz matt, Clypeus etwas verlängert, dicht gelbbraun behaart, Antenne schwarz, unten kaum bräunlich werdend, 2. Geifselglied wenig kürzer als 3. Abdomen schwarz, glänzend, Segmentbasis runzlig punktiert, Mitte gröber und sparsam punktiert, der niedergedrückte Endrand von Segment 1 fein punktiert, mit glattem Ende, von $2-5$ dicht gerunzelt und ganz matt, 6 schwarz, flach ausgerandet; Ventralsegment flach punktiert, mit blassen Endrändern, 3 flach ausgerandet, $4-5$ sehr grols, ausgehöhlt und gelbborstig gefranst. Beine schwarz, gelblich behaart, Calcar gelbbraun, Klauen braun. Beine III wie bei $N$. calida gebildet, aber schwarz, Femur stark verdickt, kantig, vor dem Ende aber ohne kleinen Zahn, Tibie weniger verbreitet und innen am Ende in braunen, gerundeten Lappen verlängert, unter welchem die Calcaria entspringen. Flügel gleichmäfsig getrübt, Rand kaum dunkler, Adern und Tegulae gelbbraun, letztere mit schwarzer Scheibe. L. $10-11 \mathrm{~mm}$, Br. $3 \frac{1}{2} \mathrm{~mm}$.

$1 \sigma^{7}$ von Asmara in Eritrea; $\mathrm{Kr}$ iste $\mathrm{n}$ se $\mathrm{n}$ leg.

Nordost-Afrika.

Nomia quadrispinosa n. sp. or.

Der $N$. calida täuschend ähnlich, aber Segment $1-5$ hellgelb gebändert, 6 schwarz, Ventralsegmente $3-4$ jederseits mit langem bewimperten Dorn.

o. Schwarz, kurz gelbbraun behaart, Kopf und Thorax ziemlich grob runzlig punktiert, glänzend, Gesicht gelbfilzig behaart, glänzend; Antenne gelb, Geifseln oben bräunlich, 2. Geifselglied $=3$; Mandibel gelb, mit rotbraunem Ende; Ränder des Mesonotum, Scutellum und das ganze Metanotum gelbfilzig. Abdomen ziemlich grob punktiert, glänzend, der niedergedrückte Endrand der Segmente 1-5 äufserst fein gerunzelt und matt, breit gelb gefärbt, 6 schwarz, mitten etwas vorgezogen, schwarz behaart; Ventralsegmente sparsam punktiert, 3-4 breit und tief ausgerandet, jederseits in feinen braunen Chitinlappen verlängert, der $2 \mathrm{~mm}$ über den Seitenrand hervorragt, also auch von oben sichtbar ist, Segmentrand und Dornlappen lang gelbbraun bewimpert, 5 ausgehöhlt, jederseits mit schwarzem Dorn, 6 konkav. Beine gelb, nur Coxa, Trochanter und Basis von Femur III schwarzbraun, gelblich behaart, Femur III verdickt, Ende unten mit kleinem Zahn, Tibie III dem Ende zu stark verbreitert, 3kantig, an der Basis aufsen mit braunem Fleck, inneres Ende 
zahnartig verlängert, unter dem Zahn die beiden Calcaria, Tarsen III am Ende mit starker Bebüschelung. Flügel gelblich getrübt, mit dunklem Rande, Adern und Tegulae gelbbraun. L. 12 mm, Br. $3 \frac{1}{2} \mathrm{~mm}$.

$\sigma^{T}$ von Delagao-Bai und Lukuledi in Deutsch-Ostafrika.

36. Nomiabrevipennis n. sp. ơ.

Der $N$. amoenula Gerst. von Ost-Afrika ähnlich, aber Scutellum unbedornt, Abdomen schwarz, Beine schwach bewehrt.

$2 \sigma^{7}$. Schwarz, weifslich behaart, Kopf und Thorax dicht runzlig punktiert, matt, Kopf viel breiter als lang, Gesicht weifsfilzig, Mandibel gelb mit rotem Ende, Metanotum weifslich befilzt, Area grob gerunzelt, glänzend. Abdomen fein punktiert, glänzend, die niedergedrückten Endränder der Segmente blafs und mit einzelnen, anliegenden weifsen Wimperhaaren bedeckt, 6 rotgelb. Ventralsegmente kahl, undeutlich punktiert, 4 gekielt. Beine schwarz, Kniee braun, Tarsenglied 1 gelb, Femur III verdickt, Tibien keulenförmig verdickt, Ende nach innen zahnartig verlängert und hier die beiden Calcar tragend, die breit und mit umgebogener Spitze sind. Flügel gelblich getrübt, Adern braun, Tegulae rotgelb. L. $6-6^{1} / 2 \mathrm{~mm}, \mathrm{Br} .1^{3} / 4 \mathrm{~mm}$.

$2 \sigma^{7}$ von Harrar, im November, K r iste $\mathrm{n}$ s e n leg. Südost-Abessinien.

37. Scapter abessinicus n. sp. or.

Wie Scr. armatus Magr., aber Trochanter ohne Zahn oder Dorn, Analsegment nicht verbreitert, Abdomen bindenlos.

$\sigma^{7}$. Schwarz, kaum weifslich behaart, Kopf grob punktiert, fast gerunzelt, Gesicht behaart, Clypeus gerunzelt, Antenne sehr kurz, schwarzbraun, die einzelnen Glieder fast so lang wie breit (bei Scr. armatus viel breiter als lang). Thorax gerunzelt, fast matt; Mesonotum und Scutellum sehr fein punktiert, Area gerunzelt, seitlich davon lang weifslich behaart. Abdomen unregelmäfsig punktiert, Segment 1 grober punktiert und wie Segment 2 glänzend, 3-7 mit braunen Endrändern und schwach gelblich behaart, 7 klein und vorgezogen, rot, ausgebuchtet und rotgelb behaart. Beine schwarz, sparsam gelblich behaart, Tarsenglied 2-5 rotgelb, Calcar schwarzbraun. Flügel hyalin mit getrübtem Rand, Adern und Tegulae schwarzbraun. L. $7 \mathrm{~mm}$, Br. $2 \mathrm{~mm}$.

$1 \sigma^{7}$ von Harrar, im Dezember, Krist en s e n leg.

Südost-Abessinien.

43. Allodape candida Sm. or.

$\sigma^{7}$ dem $q$ kaum noch ähnlich sehend, mehr wie eine kleine 
Meliturga (im $\sigma^{7}$ ). Schwarz, lang und dicht grau behaart, Kopf viel kleiner als der Thorax, fein runzlig punktiert, matt, Augen sehr grols, nehmen $2 / 3$ des ganzen Kopfes ein, im Tode rotgelb, Augenränder und Stirn mit schwarzen Haaren, Clypeus klein, Labrum fast quadratisch, Mandibel fast linear schmal, Wangen deutlich; Antenne sehr kurz, kürzer als Augenlänge, schwarz, Geifsel rotgelb, dick keulenförmig, alle Glieder 2 3 mal so breit wie lang, Geifsel kaum länger als der Schaft. Mesonotum fast glatt, glänzend, an den Rändern mit einzelnen feinen Punkten, Scutellum gröber punktiert, ebenso Mesopleuren, hintere Thoraxwand fast glatt, aber wenig glänzend. Thorax überall lang und wollig grau behaart. Abdomen höckrig runzlig punktiert, Segment 1 lang und dicht grau behaart, 2 ziemlich lang schwarz behaart, $3-5$ kurz und einzeln schwarz behaart, 6-7 lang weifslich behaart, 3-6 auf der Mitte mehr weniger graufilzig, wie beim $q$, 7 breit, mit abgestutztem Ende. Ventralsegmente fein gerunzelt, mit braunen Endrändern, lang wollig und grau behaart. Beine und Unterseite lang grau behaart, schwarz, nur Tarsenglieder 3-5 rotbraun; Beine'I stark verlängert, besonders Tibie und Tarsen, Tibie von Femurlänge, Tarsenglied 1 längér als die Tibie und fadendünn, wie die andern Tarsenglieder, Calcaria blafs. Flügel hyalin, Adern und Tegulae braun. L. $8 \mathrm{~mm}$. Br. $3^{1} / 2 \mathrm{~mm}$. $1 \sigma^{7}$ von Marienhof, Insel im Ukerewe-See, Co n r a d t s leg. Central-Afrika.

\section{Allodape foveata Sm. ơ.}

$\sigma^{7}$ wie + , aber aufser dem Clypeus auch ein Fleck daneben, ein Fleck auf den Mandibeln, das Labrum und die Vorderseite des Antennenscapus gelb, Antenne schwarz, Endglied aber zugespitzt. Calli h. oft rotbraun, Segment 7 mit einer Reihe (8-10) langer, gelber und abstehender Borsten, Tarsen gelblich, Metatarsus (III) fast weifs. L. $6 \mathrm{~mm}$, Br. $1 \frac{1}{2} \mathrm{~mm}$.

$2 \sigma^{7} 1$ o von Harrar, im November fliegend, Kristens e $n$ leg.

Südost-Abessinien.

68. Xylocopa nigripes n. sp. orㅇ.

Der $X$. scioënsis Grib. sehr nahestehend, aber Kopf, Beine und die ganze Unterseite schwarz behaart, beim or der Kopf hell behaart aber Kopf und Thorax mehr weniger schwarz behaart.

․ Schwarz, schwarz behaart, Kopf und Thorax grob punktiert, stellenweise gerunzelt, glänzend, Clypeus etwas konkav, mit kielartig erhabenen Rändern, Stirnhöcker nur schwach entwickelt. Mesonotumscheibe glatt und glänzend, Scutellum scharf- 
kantig. Thorax oben bis zu den Calli h. und das Segment 1 dicht gelb behaart. Abdomen grob und sparsam punktiert, mit schwarzbehaarten Seitenrändern, Segment 6 mit oben ausgehöhltem Enddorn in der Mitte, infolgedessen die braune Behaarung auffällt. Ventralsegmente punktiert, glänzend, lang schwarz gefranst. Beine schwarz, schwarz behaart, Klauen rotbraun, Calcar schwarz, mit roter Spitze. Flügel schwarzbraun, violett und blau schillernd, Adern, Tegulae schwarzbraun. L. $13-14 \mathrm{~mm}$, Br. $6 \mathrm{~mm}$.

$\sigma^{7}$ wie $q$, etwas grölser, aber Kopf gelb behaart, mit lang weifs behaartem Gesicht, Clypeus vorne gelb gefleckt, Antenne schwarz, unten gelbbraun, Schaft vorne gelb. Thorax lang gelb behaart, aber mit schwarz behaarter Scheibe, die dunkle Behaarung vorne bis zum Kopfe reichend. Segment 1 lang gelblich behaart, 2-3 meist nur seitlich und grünlichgelb, mitten aber wie 4-7 schwarz behaart. Ventralsegmente und Beine schwarz, lang schwarz behaart. L. $14-15 \mathrm{~mm}$, Br. $6 \mathrm{~mm}$.

$\sigma^{7}+$ in Mehrzahl von Harrar, im November fliegend, K rist en s e n leg.

Südost-Abessinien.

70. Tetralonia abessinica n. sp. oro.

Der T. albocincta Fr. aus Süd-Afrika nahestehend, aber Antenne und Beine schwarz, Segment 2 auch mit weifser Randbinde.

․ Schwarz, lang weifslich behaart, Kopf fein punktiert, Clypeus und Labrum grob runzlig punktiert, Antenne schwarz, 2. Geifselglied fast so lang wie $3+4$. Thoraxscheibe lang gelbbraun behaart, grob aber flach runzlig punktiert. Abdomen schwarz, Segmente punktiert, die breiten Endränder fast glatt, Segment 1 lang büschelig behaart, $2-4$ mit weifsfilziger Basis, $2-3$ auch mit befilztem Endrand, 4 ganz weifsgrau befilzt, 5-6 lang schwarzbraun beborstet, Analplatte schwarzbraun, kahl, kaum skulpturiert. Ventralsegmente schwarzbraun grob punktiert, rotgelb behaart, Endränder fast glatt und häutig. Beine schwarz, gelblich behaart, Scopa sehr dicht und lang, aufsen weifs, innen rotgelb, Calcar rotgelb. Flügel stark getrübt, Adern und Tegulae schwarzbraun. L. $11-11^{1} / 2 \mathrm{~mm}, \mathrm{Br} .4 \mathrm{~mm}$.

$\sigma^{7}$ wie + , Clypeusrand gelb, Mandibel schwarz, Antenne lang, schwarzbraun, unten rotbraun, erreichen fast das Abdomenende, 2. Geifselglied sehr kurz, nur $1 / 4$ des 3. erreichend; Segment 3 -5 fast ganz weifsfilzig und dadurch unter allen äthiopischen Arten auffallend, $6--7$ fast schwarzbraun behaart. Ventralsegmente fein punktiert, weifslich gefranst, Analsegment mitten gefurcht, seitlich gewulstet. L. $10 \mathrm{~mm}$, Br. $3 \frac{1}{2} \mathrm{~mm}$. 
Hochland.

$\sigma^{7}$ 우 mehrfach von Harrar, im November, Kriste n s e n leg.,

Südost-Abessinien.

T. abessinica ist vielleicht nur eine Form von $T$. albocincta, doch läfst sich darüber erst Entscheidung treffen, wenn das $\sigma^{7}$ von $T$. albocincta bekannt geworden ist.

\section{Tetralonia femorata n. sp. $\sigma^{7}$.}

Wie $T$. dentata Klg. aus Europa, aber Mesonotum grob runzlig punktiert, Mandibel schwarz und Femur mitten und hinten mit Zahn.

o7. Schwarz, lang gelblich behaart, Kopf schwach runzlig punktiert, glänzend, Clypeus gelb, Antenne schwarz, Geifselglied 2 sehr kurz, nur $1 / 6$ vom 3. erreichend, die einzelnen Glieder stark gebogen. Mesonotum und Scutellum grob runzlig punktiert. Abdomen runzlig punktiert, nur der äufserste Endrand der Segmente glatt und braun, Segment $2-3$ jederseits mit breitem, dreieckigem, gelblichem Filzfleck, auch die 3. ganze Basis befilzt, 4-6 fast ganz gelblich befilzt, 6 jederseits mit kleinem Zahn, 7 rotgelb behaart. Ventralsegment sparsam punktiert, glänzend, mit breiten, häutigen Rändern und nur seitlich gefranst, 6 mitten gefurcht, jederseits mit wulstigem Rande. Beine schwarz, lang gelblich behaart, Tarsenglied 2-4 mehr weniger rotbraun, Femur II verdickt und verkürzt, Tarsen verlängert, Femur III kaum verdickt, aber mitten und hinten mit deutlichem Zahn, Metatarsus dem Ende zu breiter werdend, innen lang rotgelb behaart. Flügel getrübt, Adern braun, Tegulae gelbbraun. L. $10 \mathrm{~mm}, \mathrm{Br} .3^{1 / 2} \mathrm{~mm}$.

$2 \sigma^{7}$ von Harrar, im Oktober, Kriste $\mathrm{n}$ s e n leg.

Südost-Abessinien.

Die ähnliche T. braunsiana Fr. $\sigma^{T}$ vom Kapland hat gelbes Labrum, Mandibel und alle Tarsen gelbbraun, Femur III ohne Zahn und Segment 6 ohne Zahn.

79. Anthophora ampliceps n. n. (für A. laticeps) var. kristenseni $\mathrm{n}$. var.

1911. A. laticeps Friese, $\sigma^{7}$ in: Zool. Jahrb. Syst. v. 30 p. 675 (nec A. (Eucara) laticeps 1905.

var. $\sigma^{7}$. Wie $A$. ampliceps, aber Clypeus ganz gelb, Nebengesicht bis zur Antennenbasis, also die untere Gesichtshälfte geschlossen gelb gefärbt; Segment $2-5$ fast schwarz behaart (kurz und nicht grau wie bei ampliceps). Dadurch die hellen Fransenbinden schärfer hervortretend. L. 10-11 mm. var. kristenseni $\mathrm{n}$. var. $\sigma^{\top}$ nicht selten bei Harar, im Oktober, Kristensen leg. Südost-Abessinien. 
80. Anthophora eritrina n. sp. or 9 .

Wie A. circulata F., aber Behaarung mehr weifslich, Segmentbinden weifs, Clypeus ausgedehnt schwarz gezeichnet.

․ Schwarz, weifslich behaart, Kopf und Thorax runzlig punktiert, fast matt, Clypeus fast schwarz, nur Ränder und Mittellinie schmal gelbweifs, Nebengesicht mit kleinem, gelbweifsem Fleck; Labrum weifslich, der Endrand und 2 grofse Basalflecke schwarzbraun, Mandibel meist gelb, Thorax oben gelbbraun, unten weifslich behaart, Behaarung des Mesonotum reichlich mit schwarzen Haaren gemischt. Abdomen punktiert, stellenweise gerunzelt, Segmentbinden $1-5$ weifs, Segment 6 mitten schwarz, seitlich weifs behaart. Ventralsegmente grofs und flach punktiert, kurz rötlich gefranst. Beine schwarzbraun, weifslich behaart, Scopa oben schwarzbraun, dann weifslich und unten rötlich, Tibie Innenseite schwarzbraun behaart, Metatarsus an der Basis weifslich behaart, Calcar fast schwarz, lang und sehr stark entwickelt. Flügel gebräunt, Adern schwarzbraun, Tegulae rotgelb. L. $10 \mathrm{~mm}$, Br. $4 \mathrm{~mm}$.

$\sigma^{7}$ wie + , aber Gesicht in gröfserer Ausdehnung gelbweifs, doch ist der Clypeus im Gegensatz zu $A$. circulata $\sigma^{7}$ gröfstenteils schwarz, infolge der grofsen Basalflecke, Labrum und Mandibel fast ganz gelb, ebenso das Nebengesicht. Analsegment (7) mit 2 vorspringenden Seitenecken, schwarz behaart. Beine II etwas verlängert, aber ohne besondere Bewimperung. L. $9^{1 / 2} \mathrm{~mm}$, Br. $3 \frac{1}{2} \mathrm{~mm}$.

$\sigma^{7}+9$ mehrfach von Asmara (Eritrea), im Juni fliegend, $q$ von Mulango (Brit. Ost-Afrika), 2 o auch von Zungaru in NordNigeria (Süd-Afrika), im November, im Mus. L on don.

Vielleicht als hellbehaarte Varietät von $A$. circulata F. aufzufassen.

81. Antophoraflaviventris n. sp. o7.

Der $A$, eritrina nahestehend, aber Gesicht dunkelgelb fast rotgelb gezeichnet, Beine II stark verlängert und Klauenglied jederseits lang schwarz bewimpert.

$\sigma^{7}$. Schwarz, lang weifslich behaart, Kopf und Thorax oben gelbbraun behaart, runzlig punktiert, fast matt. Rotgelb resp. gelb sind: Clypeus, Stirnschildchen, Nebengesicht, Labrum, Mandibelbasis und Antennenschaft vorne. Antenne ganz schwarz. Mesonotum mit glattem, glänzendem Scheibenfleck. Abdomen unregelmäfsig punktiert, Segment 1 lang gelbbraun behaart, 2-6 schwarz, aber kurz und sparsam behaart, 2-6 mit schmalen, weifsen Fransenbinden, 7 ganz schwarzbraun, 2spitzig. Ventra]segmente feiner punktiert, $3-5$ mitten eingedrückt, $3-4$ aus- 
gerandet und gelbrandig, die Vertiefungen dicht rotgelb und bürstig behaart, 5. mehr flach und gröfser, ziemlich kahl und mit ganzem Rande. Beine schwarz, fast weifs behaart, Calcar rotgelb. Beine II stark verlängert, besonders die Tarsen, Tarsenglied 1 von Tibienlänge, Klauenglied jederseits lang schwarz bewimpert, Beine III etwas verdickt, besonders die Tibie, Metatarsus leicht gebogen. Flügel getrübt, Adern braun, Tegulae rotgelb. L. $11 \mathrm{~mm}$, Br. $3 \frac{1}{2} \mathrm{~mm}$.

$2 \sigma^{7}$ von Asmara (Eritrea), im August fliegend.

Nord-Abessinien.

\section{Anthophora caerulea Friese (1905) or.}

$\sigma^{7}$ wie 9 , aber die weifse Gesichtszeichnung etwas reichlicher vorhanden, aber durchaus nicht derart wie bei den meisten Anthophora $0^{7}$, aber der Antennenschaft vorne weifs; Tibie III aufsen ganz weifs behaart und Metatarsus wie beim $q$ schwarz behaart, mit weifs behaarter Basis. L. $9-10 \mathrm{~mm}, \mathrm{Br}$. $3 \frac{1}{2} \mathrm{~mm}$.

$\sigma^{7}+$ zahlreich von Harrar, im November fliegend, Kristensen leg., + von Gheleb (Eritrea) im September, sonst von Sierra Leone, Kongo, Kamerun und Ost-Afrika bekannt; 1 o von Natal (Durban) im Kap-Museum.

\section{Epeoluskristenseni n. sp. 중.}

Wie E. tristis Sm. aus Zentral-Europa, aber Mesonotum und Scutellum ohne weifse Filzflecke, 2. Geifselglied schwarz, ơ nur Ventralsegment $4-5$ mit fast schwarzen Wimperborsten, deren Ende aufwärts gekrümmt ist.

q. Schwarz, stellenweise weifsfilzig behaart, Kopf grob und tief punktiert, Clypeus viel breiter als lang, gerunzelt, Labrum mit einzelnen groben Punkten, Antenne schwarz, 2. Geifselglied viel länger als 3. Mesonotum ohne weifse Filzflecke, höchstens mit einzelnen weifslichen Haaren, sonst grob und tief punktiert, stellenweise gerunzelt, Scutellum dicht gerunzelt, ganz matt. Weifs befilzt: Pronotum, Mesopleuren und Seiten der hinteren Thoraxwand, Area gerunzelt. Abdomen fein und ziemlich dicht punktiert, kahl, Segment $1-5$ jederseits mit grofsen oft eckigen weifsen Filzflecken; Ventralsegmente dicht und fein punktiert, 3-5 dünn mit kleinen weifsen Schuppen auf der Endhälfte, 6 grofs, fast matt und gerundet. Beine schwarz, sparsam weifsfilzig, Calcar schwarz. Flügel schwarzbraun, mit glashellen Flecken und violettem Schimmer, Adern und Tegulae schwarz, letztere punktiert, glänzend. L. $8^{1} / 2-10^{1} / 2 \mathrm{~mm}, \mathrm{Br} .3-3^{1} / 2 \mathrm{~mm}$.

$\sigma^{7}$ wie + , aber Segment 5-6 mit ganzer weifser Filzbinde; Ventralsegmente $2-3$ mit weifs beschuppter Endhälfte, $4-5$ mit 
fast schwarzen Wimperborsten, deren Ende nach oben umgebogen ist, Tarsenglied 1 an allen Beinpaaren lang braun beborstet. L. $7^{1} / 2-10 \mathrm{~mm}$, Br. $2^{1 / 2}-3^{1 / 2} \mathrm{~mm}$.

$\sigma^{7}+$ mehrfach bei Harrar, im November, $\mathrm{K}$ riste $\mathrm{n}$ s e n leg. Südost-Abessinien.

Wohl Schmarotzer von Colletes abessinicus Fr.

\section{Eriades filicornis n. sp. or.}

Durch die langen, dünnen Antennen auffallend, deren Glieder fast zweimal so lang wie breit sind.

○. Schwarz, stellenweise weifs behaart, Kopf und Thorax grob runzlig punktiert, fast höckerig, glänzend, Gesicht lang weifs behaart; Antenne fadenförmig dünn, lang, erreichen das Thoraxende, 2. Geifselglied kurz, knopfförmig wie das 1., das 3. und folgende doppelt so lang wie breit. Scutellum mit einzelnen und gröberen Punkten; Area grob gerunzelt, horizontale Zone scharf gerandet. Abdomen fast runzlig punktiert, Segment 1 sehr grob und tief punktiert, 2 weniger grob, 3 und folgende mehr gerunzelt und feiner, 1-2 mit feinen, weifsen Fransenbinden, 5-6 schwach gelblich behaart, 6 bogig gerundet, mitten ausgeschnitten; Ventralsegmente punktiert, stark glänzend, 3 und folgende verborgen (gelblich gefärbt). Beine schwarz, weils behaart, Tarsenglied 3-5 rotgelb, Calcar bleich. Flügel fast hyalin, mit getrübtem Endrande, Adern und Tegulae schwarz. L. $5 \mathrm{~mm}$, Br. $1 \frac{1}{2} \mathrm{~mm}$.

$2 \sigma^{\top}$ von Harrar, im November, Kristensen leg. Südost-Abessinien.

Am besten bei E. argentatus Gerst. stehend, aber grölser.

$$
\text { Eriades longicornis n. sp. or. }
$$

Wie E. filicornis (die vorhergehende) mit langen Antennen, aber die Glieder nur $1 \frac{1}{2}$ mal so lang wie breit und die Flügel gebräunt.

$\sigma^{7}$. Schwarz, aber sparsam gelblich behaart, Kopf und Thorax runzlig punktiert, matt. Antenne lang, erreicht das Thoraxende, Geifselglied $2=1,3$ und folgende $1 \frac{1}{2}$ mal so lang wie breit. Scutellum und Mesonotumscheibe tief punktiert; Area fast glatt, glänzend, obere horizontale Zone längsriefig. Abdomen tief punktiert, etwas glänzend, Segment 1-2 nur jederseits gefranst, 3-6 spärlich behaart, 6 gekielt, fast ganzrandig, jederseits vor dem Endrand leicht eingedrückt. Ventralsegment 1-2 fein runzlig punktiert, matt, 3 und folgende rotgelb und verborgen liegend. Beine schwarz, Femur rotbraun, weifs behaart, 
nur Klauen braun. Flügel stark gebräunt, Adern und Tegulae braun. L. $5 \mathrm{~mm}, \mathrm{Br} .1 \frac{1}{2} \mathrm{~mm}$.

$1 \sigma^{7}$ von Kigonsera.

Deutsch-Ostafrika.

\section{Eriades abessinicus n. sp. or.}

Grolse Art bei E. freygessneri Schlett. stehend, aber Segmente fast ohne Fransenbinden, mit kurzen Antennen und fast glatter Area.

$\sigma^{7}$. Schwarz, sparsam greis behaart, Kopf und Thorax grob punktiert, fast gerunzelt, Gesicht eckig, weifslich behaart, Antenne kurz, erreicht nur die Flügelbasis, 2. Geifselglied $=3,3$ und folgende etwas länger als breit. Thorax ziemlich lang behaart, Area glatt und glänzend, obere Zone längsriefig und hinten gerandet. Abdomen grob punktiert, glänzend, Segment 1-2 sehr sparsam weifs gefranst, $3 \mathrm{kahl}, 4-6$ weifslich behaart, 6 fast ganzrandig, jederseits vor dem Ende eingedrückt. Ventralsegmente punktiert, kahl. Beine schwarz, weifslich behaart, Calcar bleich. Flügel fast hyalin, Radialzelle gebräunt, Adern fast schwarz, Tegulae glatt, poliert und schwarz. L. $6-7 \mathrm{~mm}$, Br. $2 \mathrm{~mm}$.

$2 \sigma^{7}$ von Harrar, im November, Kristensen leg.

Südost-Abessinien.

\section{Eriades truncorum L.}

Von Eriades truncorum liegen $3 \sigma^{\pi}$ von Harrar vor, wo sie im November fliegen. Die Tierchen stimmen morphologisch und nach der Gröfse vollkommen mit $E$. truncorum überein, nur die Behaarung ist rein weils, anstatt gelblich.

\section{Megachile (Eumegachile) bilobata n. sp. or 9 .}

Wie $M$. antinorii, aber Segment 1 und 6 auch rot behaart, o Clypeus ganz abweichend geformt, jederseits in ein grofses, breites Horn verlängert.

9. Schwarz, schwarz behaart, Kopf und Thorax dicht runzlig punktiert, glänzend, Clypeus quer und jederseits mit breitem abstehenden Horn, das vorne gerundet, glatt und glänzend ist, in der Ausrandung zwischen den Hörnern mit kleinem Zahn, die Hörner überragen die Mandibeln bis zur Mitte; Mandibel an der Spitze mit 3 Zähnen und mitten am Innenrand mit grofsem, breitem, stumpfem Zapfen, der vorne 2höckrig ist. Mesonotum lang schwarz behaart, Scutellum und Area meist mit einigen rotgelben Haaren, Area fein gerunzelt und gegen die gröbere Umgebung abstechend. Ventralsegmente schwarz, Scopa ganz schwarz, Beine schwarz, schwarz behaart, Metatarsus kaum von 
Tibienbreite, Calcar rotbraun. Flügel schwarzbraun, bläulich schimmernd, Adern und Tegulae schwarzbraun. L. 17-18 mm, Br. $5-5^{1 / 2} \mathrm{~mm}$.

$\sigma^{7}$ wie $\$$, aber Clypeus, Stirnschildchen, Kopfunterseite und Beine I lang weifslich behaart; Thorax mehr oder weniger braun behaart, Segment 1-6 rot behaart, Analsegment ausgebuchtet, wie bei $M$. antinorii ; Beine einfach. L. $13-15 \mathrm{~mm}$, Br. $4-5 \mathrm{~mm}$.

q var. - Segment 6 schwarz behaart, - var. commixta n. var.

Einige $\sigma^{7} q$ von Harrar, im November fliegend, Kristensen leg. (auch die var. commixta).

\section{Megachile menelikin. sp. 우.}

Wie $M$. antinorii, aber noch gröfser und gröfste afrikanische Megachile-Art, Segment 5-6 schwarz behaart, $2-5$ mit rotgelben Fransenbinden.

․ Schwarz, schwarz behaart, morphologisch wie $M$. antinorii gebaut; Mandibel verlängert und mit 4 spitzen Zähnen, der Dorn vorne an der Clypeusmitte ist stumpf, breit, wenig vorragend und 2höckrig. Thorax schwarz bis schwarzbraun behaart, Segment 1-4 rot hehaart, 5-6 schwarz behaart, aber Segment $2-5$ mit deutlichen und ziemlich breiten rotgelben Fransenbinden, Scopa schwarz, Metatarsus von Tibienbreite. Flügel schwarzbraun mit blauem Schimmer. L. $24-25 \mathrm{~mm}$, Br. $8 \mathrm{~mm}$.

2 ㅇ von Süd-Abessinien.

Gröfste Megachile-Art von Afrika, die wohl nur von einigen Exemplaren der $M$. maxillosa erreicht wird; der mächtige Kopf läfst sie gut von $M$. antinorii unterscheiden.

Megachile cognata var. claripennis n. var. ㅇ.

Wie $M$. cognata Sm. (Kapland, Ukami), aber Flügel hell, stark gelblich getrübt, Adern gelbbraun, Tegulae braun. L. $16 \mathrm{~mm}$, Br. $5 \mathrm{~mm}$.

1 \& von Lukuledi in Deutsch-Ostafrika.

119. Megachile abessinica n. sp. 중.

Wie M. filicornis Fr. aus Ost-Afrika, aber Segment 6 schwarz behaart und Flügel schwarzbraun, + mit schwarzer Scopa, ơ Tarsen I auch verbreitert, weifs, aber nach vorne noch lappig erweitert, von Tibienbreite und nach hinten weifs gefranst.

․ Schwarz, lang und dicht schwarz behaart, Kopf und Thorax dicht, aber flach punktiert, stellenweise gerunzelt, Clypeus flach, mit glattem Mittelfeld, stumpf, mitten ausgerandet, Antenne schwarz, 2. Geifselglied $=3$, Endglied abgeplattet, so lang wie das vorletzte. Abdomen schwarz, kurz oval, flach punktiert, lang 
rot behaart, Segment 1 gröfstenteils schwarzbraun behaart, 6 schwarz behaart, Scopa lang und schwarz. Beine schwarz, schwarz behaart, Calcar schwarz, der äufsere länger, stumpf und geknöpft am Ende, Metatarsus fast breiter als die Tibie III. Flügel schwarzbraun, mit violettem Schimmer, Adern schwarzbraun, Tegulae schwarz. L. $16-18 \mathrm{~mm}$, Br. $6 \mathrm{~mm}$, als eine auffallend breite Art.

$\sigma^{7}$ wie $q$, aber mehr schwarzbraun behaart, Gesicht lang weifsgelb und seidenglänzend behaart; Antenne schwarz, 2. Geifselglied etwas kürzer als 3. Segment 1 fast ganz rot, wie die übrigen Segmente behaart, $6 \mathrm{schwarz}$ behaart, mitten tief ausgerandet. Ventralsegmente flach, sparsam und flach punktiert, dünn weifslich gefranst, mit häutigen, oft abstehenden Rändern. Beine 1 rotgelb, schwarz gefleckt, Tarsen I weifs, verbreitert und nach vorne lappig erweitert, nach hinten lang und dicht weifs bewimpert, von Tibienbreite. L. $15-16 \mathrm{~mm}, \mathrm{Br} .5^{1 / 2} \mathrm{~mm}$.
$\sigma^{7}+$ mehrfach von Harrar, im November fliegend, Kristen- s e n leg.
Südost-Abessinien.

120. Megachile harrarensis n. sp. $\sigma^{7}$.

Der M. rufa Fr. $\sigma^{T}$ aus Ost- und Süd-Afrika ähnlich, aber Segment 5-6 schwarz behaart, Tarsen I nicht verbreitert.

$\sigma^{7}$. Schwarz, dicht gelbbraun behaart, Kopf und Thorax dicht runzlig punktiert; Antenne schwarz, 2. Geifselglied $=3$., Endglied nach hinten breit lappig erweitert, Abdomen lang und dicht gelbbraun behaart, Segment 5-6 schwarz, 6 ausgehöhlt, lappig vorstehend, mitten ausgerandet; Ventralsegmente einzeln und flach punktiert, mit häutigem Rand. Beine schwarz bis schwarzbraun, verdickt und gekrümmt, Calcar rotgelb; Beine I innen rotgelb, Tarsen gelblich, kaum sichtbar verbreitert, nach hinten aber lang weifs bewimpert, Tarsen II lang weifs behaart, Metatarsus verkürzt, fast von Tibienbreite, aber nur so lang wie breit (eckig), Tarsenglied 2-4 der Beine III lappig verbreitert. Flügel fast hyalin, mit getrübtem Rande, Adern und Tegulae braun. L. $13 \mathrm{~mm}$, Br. $5 \mathrm{~mm}$.

$2 \sigma^{7}$ von Harrar, im November, Kristensen leg.

Südost-Abessinien.

132. Anthidium cordatum var. rufescens n. var. or $\sigma^{7}$.

var. $\sigma^{\top} q$ wie A. cordatum, aber gröfser, Thorax schwarz, nur beim $\sigma^{7}$ mit winzig kleinen gelben Flecken; Stirn und Stirnschildchen, beim $q$ auch noch die Mandibel ganz schwarz; Abdomenseiten und Ende kurz rotgelb behaart. Beine rotgelb, Femur 
schwarz; Flügel gebräunt und bläulich schimmernd. L. $10 \mathrm{~mm}$, Br. $4^{1} / 2 \mathrm{~mm}$.

var. $r u f e s c e n s$ n. var.

$\sigma^{\top} ㅇ$ von Harrar, im November, Kristensen leg.

Südost-Abessinien.

Anthidium cordatum var. salamensen. var. 우.

var. 우 wie $A$. cordatum, aber die gelben Zeichnungen auf Kopf und Thorax fast elfenbeinweifs, so die Seiten des Mesonotum breit weifslich, Scutellumecken und ein breiter Streifen unterhalb der Tegulae-Abdomen rotgelb, nur Segment 1 mitten schwarz, 1-5 mit breiten, gelben Seitenflecken. Ventralsegmente gelb, weifs behaart. Flügel getrübt mit breitem, dunklem Endrand, Adern und Tegulae braun, letztere vorne mit weifsem Fleck. L. $81 / 2-9 \mathrm{~mm}$, Br. $4 \mathrm{~mm}$.

1 우 von Dar-es-Salaam, Deutsch-Ostafrika.

var. salamense n. var.

Anthidium cordatum ist offenbar eine sehr variable Spezies, was die Ausdehnung der Gelbfärbung betrifft. Alle mir vorliegenden Exemplare (14) weichen voneinander ab, sowie sie aus einer andern Gegend stammen. Solche Gegenden sind: Capland, Algoa-Bai, Port Natal, Delagoa-Bai, Mosambique, Dar-es-Salaam und Harrar. Die Exemplare von der Algoa-Bai und Mosambique stimmen am besten mit der Beschreibung von Smith überein. Die Form, Skulptur und beim or das 3zackige Analsegment lassen die Art aber leicht erkennen.

133. Anthidium eritrinum n. sp. ㅇ.

Dem A. truncatum ähnlich, aber Scutellum gerundet, Mandibel stumpf und Segment 2-6 mit ganzen gelben Binden.

\%. Schwarz, kurz gelblich bis rotgelb behaart, Kopf und Thorax grob runzlig punktiert, Gesicht bis zu der Antennenbasis gelb, Clypeus gerundet, vorne mit 5 schwarzen Höckern, Mandibel rot, kurz und breit, nur an der Spitze mit 2 kurzen Zähnen, sonst ganzrandig, also ohne die verlängerten und lang und scharf bezahnten Mandibel des wolleschabenden A. truncatum. Antenne rotbraun, Hinterrand des Kopfes jederseits gelb gefleckt. Thorax schwarz, Calli hum. nach vorne schildartig und häutig verlängert, mit gelber Scheibe. Scutellum gerundet, nach hinten verlängert, mitten ausgerandet. Abdomen grob punktiert, Segment 1 jederseits mit breitem, gelbem Fleck, 2-6 mit ziemlich breiter gelber Binde auf der Basalhälfte; Ventralsegmente rotgelb, Scopa gelblich. Beine gelb, Basalteil mehr weniger schwarzbraun, Tarsen rotgelb. Flügel gebräunt, Adern schwarzbraun, Stigma rot, 
Tegulae sehr grofs, rotgelb mit schwarzbrauner Basis. L. $6 \mathrm{~mm}$, Br. $2^{1 / 2} \mathrm{~mm}$.

o von Gheleb, im September, Kristensen leg.

Eritrea (Nordost-Afrika).

142. Omachtes nigripes n. sp. ơ (Olim „O. carnifex Gerst." determ.)

Nach der Beschreibung der O. carnifex am ähnlichsten, aber Beine schwarz und Flügel gelblich getrübt mit braunem Rande.

9. Schwarz, kurz schwarzbraun, fast filzig behaart, Kopf und Thorax ziemlich grob runzlig punktiert, aber stellenweise glatt und glänzend (wie an den Mesopleuren und am Scheitel des Kopfes), Kopf etwas breiter als lang, Gesicht weifsfilzig behaart, auch das Labrum, Mandibel mit rotem Ende, Antenne schwarz, 2. Geifselglied so lang als $3+4,3-4$ doppelt so breit wie lang, 5 und folgende fast quadratisch. Mesonotum uneben, Scutellum 2 beulig, Area dicht gerunzelt, matt, nicht auffallend. Abdomen sehr undeutlich punktiert, fast glatt, glänzend und fast kahl, stellenweise mit kurzer, gelbbrauner Seidenbehaarung, sonst ganz rot gefärbt und nur der Endrand vom Segment 6 schwarz. Ventralsegmente rotgelb, kahl, 5 stumpf erhaben, halbrund ausgerandet, an der Ausrandung gelbfilzig behaart, in der Ausrandung ragt das 6. als zweizinkige Gabel hervor. Beine schwarz, stellenweise kurz gelbfilzig, Calcar rot. Flügel gelblich getrübt, mit dunklem Endrand, Adern gelbbraun, Tegulae schwarz. L. $11 \mathrm{~mm}$, Br. $3 \mathrm{~mm}$.

$\sigma^{7}$ wie $q$, nur Labrum jederseits mit langem, braunem Borstenbüschel, Abdomen rot, Segment $6-7$ ganz rot, 7 verlängert, etwas verjüngt mit abgerundetem Ende, der erhabene Rand schwarzbraun; Ventralsegmente mit gelblichen Haaren spärlich besetzt. Beine schwarz, kaum behaart. L. $9^{1} / 2 \mathrm{~mm}, \mathrm{Br} .2^{1 /} / 4 \mathrm{~mm}$.

$\sigma^{7}+$ von Eritrea und $1 q$ von Usambara, Deutsch-Ostafrika.

(Die Fundorte bei $O$. carnifex in meinen "Bienen Afrikas" p. 437 beziehen sich auf diese Art.)

2. Discoidalquerader stöfst auf die 2. Kubitalquerader.

\section{Omachtes capensis n. sp. ㅇ.}

Dem O. carnifex offenbar verwandt, aber Segment 4-6 schwarzbraun, Flügel hell, milchig getrübt, Antenne braun.

9. Schwarz, schwarz bis schwarzbraun behaart, Kopf dicht und ziemlich grob runzlig punktiert, fast matt, nur am Stirnschildchen mit glatter Stelle, Kopf fast zweimal so breit wie lang, Mandibel schwarzbraun, mit roter Endhälfte, Antenne braun bis rotbraun, 2. Geifselglied etwas länger als 3 ; Area nicht auf- 
fallend, seitwärts davon mit kurzen, greisen Haaren. Abdomen fast glatt und kahl, kaum erkennbar punktiert, Segment 1-3 und Basis von 4 rot bis rotgelb, Rest schwarzbraun, 5-6 braun behaart. Ventralsegmente 1-4 kaum punktiert, fast glatt, glänzend, 4-5 grob punktiert, fast matt, 5 stumpfkielig erhaben, braunborstig behaart, 6 als 2spitziger Stempel vorragend. Beine rot bis rotbraun, kurz greis behaart, Calcar rot. Flügel fast hyalin, milchig getrübt, Adern gelbbraun, Tegulae rotgelb. L. $7 \mathrm{~mm}$, Br. $1^{3} / 4 \mathrm{~mm}$.

1 우 vom Kapland.

Flügelgeäder wie bei Pasites (Omachtes) villosus, die 2. Discoidalquerader mündet vor der 2. Kubitalquerader in die 2. Kubitalzelle.

Omachtes (Pasites olim) villosus Friese (1909). ㅇ.

$q$ wie das $\sigma^{7}$, aber grölser, Segment $4-6$ schwarz, hintere Thoraxwand schwarz behaart, alle Beine schwarz. Flügel etwas stärker gebräunt. L. $8 \mathrm{~mm}, \mathrm{Br} .2^{1} / 4 \mathrm{~mm}$.

$1 q$ von Kapstadt, $1 q$ sehr grofs $=\mathrm{L} .10 \mathrm{~mm}, \mathrm{Br} .2^{1 / 2} \mathrm{~mm}$ von Java (?!).

2. Discoidalquerader mündet vor dem Ende der 2. Kubitalzelle.

143. Omachtes abessinicus n. sp. ㅇ.

Dem Pasites maculatus Jur. var. brunneus Friese aus Turkestan täuschend ähnlich, aber dem Omachtes histrio Gerst. verwandt, nur Thorax meist rotbraun, Abdomen ganz rot.

․ Rot bis rotgelb weifsfilzig behaart, Kopf schwarz, nur Mundgegend rötlich, Kopf und Thorax punktiert, etwas glänzend, Gesicht weifsfilzig, Clypeusendrand rot, Wangen, Labrum, Mandibel und Unterseite des Kopfes rot, Antenne braun, Schaft und Geifselglied 1-2 rot. Mesonotum braun, Pronotum und Ränder des Mesonotum weifsfilzig, Scutellum grob punktiert, rot und schwach 2 beulig, Mesopleuren dicht weifs befilzt, ebenso die hintere Thoraxwand. Abdomen rot bis rotgelb, fein punktiert, glänzend, Segment 1 weifshaarig, jederseits vor dem Endrand mit weifsem Filzfleck, $2-5$ mit weifsfilzig bandiertem Endrand, 6 rot. Ventralsegmente kaum punktiert, fast glatt, 2-4 mit weifsfilzigem Endrand, 5 wie bei Pasites stumpfkielig erhaben, das schmale 6. umschliefsend. Beine rotgelb, weifs befilzt, Calcar bleich. Flügel hyalin, mit schmalem, braunem Endrand, Adern braun, Tegulae gelbbraun. L. $6 \mathrm{~mm}, \mathrm{Br} .1^{3} / 4 \mathrm{~mm}$.

1 o von Eritrea.

$\mathrm{Zu}$ den Arten von Omachtes ist auch Pasites villosus Friese zu stellen, nachdem ich das $q$ dazu aus dem Kapland erhalten habe. - Ferner ist zu bemerken, dals das Flügelgeäder von $O$.

Deutsche Entomol. Zeitsehrift 1915. Heft III. 
histrio, villosus mit demjenigen von Pasites maculatus, Ammobates punctatus und Caesarea sanguinea übereinstimmt und das von $O$. nigripes, graenicheri und $O$. abessinicus abweicht, indem die 2. Discoidalquerader auf die 2. Kubitalquerader stölst. Vielleicht werden die Omachtes-Arten am besten bei Pasites untergebracht? - Der Wunsch mancher Autoren, für Omachtes Gerst. (1869) den Namen Morgania Sm. (1854) einzusetzen, lälst sich nicht erfüllen, da Morgania Sm. ohne Beschreibung und Diagnose aufgestellt wurde.

\section{Omachtes rufipes n. sp. ㅇ.}

In der Form dem 0 . nigripes ähnlich, aber breiter gebaut, Abdomen ganz schwarz, Beine rot.

․ Schwarz, stellenweise schwarz behaart, Kopf und Thorax dicht und grob runzlig punktiert, fast matt, Kopf viel breiter als lang, Clypeus vorne gerade abgestutzt und mit aufgeworfenem Endrand, Labrum quadratisch, etwas zugespitzt und rot mit schwarzer Scheibe, Mandibel rot, mit schwarzer Basis und schwarzem Ende, sichelförmig ohne Zähne; Antenne schwarz, mit rotem Schaft und rotbraunem Geifselglied 1-2, 2. Geifselglied etwas gröfser als 3, 3.-4. und folgende fast quadratisch. Mesonotum uneben, stellenweise höckerig, gerunzelt, Scutellum stark 2 höckerig, Area klein und fein gerunzelt, mit tiefer Mittelfurche. Abdomen äufserst fein und dicht skulpturiert, daher fast matt, Segmentränder nur sparsam fein punktiert, daher glatt und glänzend erscheinend, 4-6 mit einzelnen nach hinten liegenden schwarzbraunen Haaren, 6. braun bebüschelt. Ventralsegmente undeutlich skulpturiert mit fast glatten, kahlen Endhälften, Basis meist mit anliegenden schwarzbraunen Haaren, 5 rotbraun, weifslich befilzt, stumpfwinklig erhaben, ausgerandet, in der tiefen Ausrandung ragt das schmale, rote, 2spitzige Segment 6 hervor. Beine rot, sparsam weifslich behaart, mit einzelnen schwarzen dicken Borsten, besonders an der Aufsenseite, Calcar rotbraun, Flügel gebräunt, Ader braun, Tegulae rot. L. $10 \mathrm{~mm}$, Br. $3 \mathrm{~mm}$.

1 ㅇ von Zeerust (Transvaal), J e n s e n leg.

2. Discoidalquerader mündet vor der 2. Kubitalquerader.

Omachtes graenicheri Br. var. jensenïn. var. ㅇ.

Wie O. (Ammobates) graenicheri Brauns, aber viel kleiner, Ventralsegmente kahl, ohne weilse Filzbehaarung. L. $9 \mathrm{~mm}$, Br. $2^{1} / 2 \mathrm{~mm}$ (graenicheri mifst L. $12 \mathrm{~mm}$, Br. $4 \mathrm{~mm}$ ).

1 o von Zeerust - Transvaal - J e n s e n leg.

Bei O. graenicheri wie bei jenseni stölst die 2. Discoidalquerader auf die 2. Kubitalquerader. 


\section{$2 \mathrm{BHL}$ Biodiversity Heritage Library}

Friese, Heinrich. 1916. "Zur Bienenfauna von Abessinien." Deutsche entomologische Zeitschrift 1915, 265-298. https://doi.org/10.1002/mmnd.191519150310.

View This Item Online: https://www.biodiversitylibrary.org/item/110232

DOI: https://doi.org/10.1002/mmnd.191519150310

Permalink: https://www.biodiversitylibrary.org/partpdf/49410

\section{Holding Institution}

California Academy of Sciences

\section{Sponsored by}

California Academy of Sciences Library

\section{Copyright \& Reuse}

Copyright Status: Public domain. The BHL considers that this work is no longer under copyright protection.

This document was created from content at the Biodiversity Heritage Library, the world's largest open access digital library for biodiversity literature and archives. Visit BHL at https://www.biodiversitylibrary.org. 